\title{
QUASIPERIODICITY AND CHAOS IN THE NONLINEAR EVOLUTION OF THE KELVIN-HELMHOLTZ INSTABILITY OF SUPERSONIC ANISOTROPIC TANGENTIAL VELOCITY DISCONTINUITIES
}

\author{
$\mathrm{BY}$ \\ S. ROY CHOUDHURY AND KEVIN G. BROWN \\ Department of Mathematics, University of Central Florida, Orlando, FL
}

\begin{abstract}
A nonlinear stability analysis using a multiple-scales perturbation procedure is performed for the instability of two layers of immiscible, strongly anisotropic, magnetized, inviscid, arbitrarily compressible fluids in relative motion. Such configurations are of relevance in a variety of astrophysical and space configurations. For modes near the critical point of the linear neutral curve, the nonlinear evolution of the amplitude of the linear fields on the slow first-order scales is shown to be governed by a complicated nonlinear Klein-Gordon equation. The nonlinear coefficient turns out to be complex, which is, to the best of our knowledge, unlike previously considered cases and leads to completely different dynamics from that reported earlier. Both the spatially dependent and space-independent versions of this equation are considered to obtain the regimes of physical parameter space where the linearly unstable solutions either evolve to final permanent envelope wave patterns resembling the ensembles of interacting vortices observed empirically, or are disrupted via nonlinear modulation instability. In particular, the complex nonlinearity allows the existence of quasiperiodic and chaotic wave envelopes, unlike in earlier physical models governed by nonlinear Klein-Gordon equations. In addition, numerical diagnostics reveal the onset of chaos as a consequence of modulation of the external magnetic field.
\end{abstract}

1. Introduction. The Kelvin-Helmholtz instability caused by tangential velocity shear in homogeneous plasmas is of interest in investigating a variety of space, astrophysical, and geophysical situations involving sheared plasma flows. Configurations, where relevant, include the interface between the solar wind and the magnetosphere (Sen, 1965; Southwood, 1968; Southwood, 1974; Bridge et al., 1979; Ness et al., 1981; Pu and Kivelson, 1983), coronal streamers moving through the solar wind, the boundaries between adjacent sectors in the solar wind (Parker, 1963; Sturrock and Hartle, 1966; Jokipii and Davis, 1969), the structure of the tails of comets (Dobrowolny and D'Angelo,

Received February 18, 2000 and, in revised form, October 5, 2000.

2000 Mathematics Subject Classification. Primary 76E20, 76E25. 
1972; Ershkovich et al., 1972; Ershkovich and Chernikov, 1973; Brandt and Mendis, 1979), and the boundaries of the jets propagating from the nuclei of extragalactic double radio sources into their lobes (Turland and Scheuer, 1976; Blandford and Pringle, 1976; Begelman et al., 1984).

Early investigations of the Kelvin-Helmholtz instability were concerned with the instability caused by a tangential velocity discontinuity or jump (or vortex sheet) in incompressible and compressible fluids and plasmas (Landau, 1944; Fejer, 1964; Sen, 1964; Miles, 1957; Gerwin, 1968).

The unmagnetized vortex sheet is found to be unstable at all wavenumbers for modes sufficiently transverse to the zero-order flow, or for modes along the flow with Mach number less than $2 \sqrt{2}$. In the presence of a magnetic field parallel to the flow, the instability of the incompressible vortex sheet is completely stabilized unless the velocity discontinuity exceeds twice the Alfvén speed. A magnetic field transverse to the flow has no effect on the instability.

Lerche (1966) emphasized the importance of considering the finite thickness of the shear layer. The linear Kelvin-Helmholtz instability of shear layers (a region of finite width over which the velocity change occurs) for flows with a subsonic velocity change was considered by Chandrasekhar (1981). An incompressible shear layer having a "hyperbolic tangent" profile was considered by Michalke (1964). He found a criterion $k L<2$ for instability, with $k$ being so that short wavelength modes were stabilized for the finite width velocity shear. The stability characteristics of finite width unmagnetized shear layers have been considered by several authors (Blumen, 1970; Blumen et al., 1975; Ray, 1982; Miura and Pritchett, 1982; Roy Choudhury and Lovelace, 1986). The finite width shear layers exhibit unstable traveling wave modes satisfying radiation boundary conditions. These modes are absent for the unmagnetized vortex sheet, and present for the magnetized vortex sheet in a very small range of Mach numbers. The presence of the traveling wave modes means that the finite width layer is unstable at all Mach numbers. In addition, standing wave solutions analogous to the "warping" modes which occur for the vortex sheet are also present at long wavelengths and small values of the Mach number. Magnetized shear layers described by the MHD formalism have been considered for a linear velocity profile layer (Roy Choudhury, 1986; Ray and Ershkovich, 1983; Roy Choudhury and Lovelace, 1984) with both standing and traveling wave solutions, and for a hyperbolic tangent velocity profile (Miura and Pritchett, 1982) for only standing wave modes. A magnetic field parallel to the flow is found to stabilize both classes of modes. Computer simulation studies of the Kelvin-Helmholtz instabilities of planar, magnetized shear layers (Nepveu, 1980; Tajima and Leboeuf, 1980; Miura and Pritchett, 1982; Prichett and Coroniti, 1984; Miura, 1984) and of cylindrical axisymmetric jets (Norman et al., 1982) have also been carried out. Velocity shear of zero and finite thickness has also been considered in anisotropic plasmas (Talwar, 1965; Roy Choudhury and Patel, 1985). The results are analogous to the MHD case with larger instability growth rates.

The nonlinear development of the incompressible Kelvin-Helmholtz instability has been studied previously by Drazin (1970), Nayfeh and Saric (1971, 1972), and, in comprehensive fashion, by Weissman (1979). 
The comprehensive nonlinear treatment of Weissman, as well as other early work on weakly nonlinear evolution of the amplitudes of linear fields in thermal convection (Newell and Whitehead, 1969), plane Poiseuille flow (Stewartson and Stuart, 1971), a buckling problem in elasticity (Lange and Newell, 1971), and baroclinic flow (Pedlosky, 1970, 1972) have been organized into a comprehensive framework by Gibbon and McGuinness (1981) (also see Dodd et al., 1982). The nonlinear analysis divides into two distinct categories. For the first category of "dissipative" instabilities (for example, cases where viscosity, diffusion, or other damping effects play a major role), the linear dispersion relation is a complex function of the frequency $\omega$ and wavenumber $k$. The neutral $\left(\omega_{i}=0\right)$ curve in the $(k, \mu)$ plane for $\mu$ some parameter of interest in the particular problem (e.g., the Mach number in the case of Kelvin-Helmholtz instability) has a minimum at a critical wavenumber $k=k_{c}$, where the onset of linear instability first occurs. The weakly nonlinear evolution of the amplitude $A$ (of the linear fields) occurs on slow second-order time and first-order space scales $T_{2}$ and $\bar{Z}$ and is governed by the canonical GinzburgLandau (Newell-Whitehead) equation,

$$
\begin{gathered}
\frac{\partial A}{\partial T_{2}}= \pm \alpha_{1} A-\beta_{1} \frac{\partial^{2} A}{\partial \bar{X}^{2}}+\gamma_{1} A|A|^{2}, \\
\bar{Z}=Z_{1}-c_{g} T_{1},
\end{gathered}
$$

with $c_{g}=\frac{d \omega}{d k}$ being the group velocity of the linearly unstable solutions. The nonlinear evolution of $A$ under this equation has been comprehensively discussed by Lange and Newell (1974) and Newell (1974). By contrast, the second category of instabilities is the "dispersive" type and occurs when no dissipation is present. For this case, the linear $\omega-k$ dispersion relation is real, with roots occurring in complex conjugate pairs

$$
\omega=\omega_{r} \pm i \omega_{i}
$$

For this case, the weakly nonlinear evolution of the linear amplitude $A$ always occurs on slow first-order time (instead of second-order time as for the "dissipative" instabilities) and space scales $T_{1}$ and $Z_{1}$, being governed by either the canonical " $A B$ equations"

$$
\begin{gathered}
\left(\frac{\partial}{\partial T_{1}}+c_{1} \frac{\partial}{\partial Z_{1}}\right)\left(\frac{\partial}{\partial T_{1}}+c_{2} \frac{\partial}{\partial Z_{1}}\right) A= \pm \alpha A-\beta A B, \\
\left(\frac{\partial}{\partial T_{1}}+c_{2} \frac{\partial}{\partial Z_{1}}\right) B=\left(\frac{\partial}{\partial T_{1}}+c_{1} \frac{\partial}{\partial Z_{1}}\right)|A|^{2},
\end{gathered}
$$

or a canonical nonlinear Klein-Gordon equation

$$
\left(\frac{\partial}{\partial T_{1}}+c_{1} \frac{\partial}{\partial Z_{1}}\right)\left(\frac{\partial}{\partial T_{1}}+c_{2} \frac{\partial}{\partial Z_{1}}\right) A=G A-\bar{N} A|A|^{2} .
$$

Gibbon and McGuinness (1981) give several physical examples leading to one or the other of these equations. The nonlinear analysis of the incompressible Kelvin-Helmholtz instability by Weissman led to the nonlinear Klein-Gordon evolution above. The " $A B$ equations" are completely integrable by the Inverse Scattering (or Spectral) Transform (Ablowitz and Segur, 1981; Dodd et al., 1982) and have been considered by Gibbon et al. (1979) and Gibbon and McGuinness (1981). The nonlinear Klein-Gordon equation, 
however, is not completely integrable, and its solutions have been discussed by Weissman (1979), Murakami (1986), and Parkes (1991).

Recently, there has been a considerable amount of work on anisotropic shear layers which are of great relevance in magnetospheric situations (see Brown and Choudhury, 1997, 1998 and references therein). In particular, a model due to Abraham-Shrauner (1973) with generalized anisotropy (different plasma pressures in different directions) has been employed to significantly generalize earlier work in this area. In this paper, we consider the weakly nonlinear evolution of the supersonic (or strongly compressible), anisotropic magnetized Kelvin-Helmholtz instability based on this model. Given the broad relevance of this instability in a variety of astrophysical and space settings, such an analysis is necessary to understand the large-scale behavior of these flows. As a first step, we will consider a spatially homogeneous equilibrium here. The governing equation turns out to be a nonlinear Klein-Gordon equation, but with a twist. The nonlinear coefficient $\bar{N}$ turns out to be complex, leading to dynamical behaviors very different from those possible for $\bar{N}$ real, including the possibility of quasiperiodic and chaotic wave envelopes. To the best of our knowledge, this has not been considered previously.

We also examine the time dependence of the solution brought on by an additional alternating forcing magnetic field corresponding to $\delta \cos \left(\Omega_{0} t\right)$, where the control parameter $\delta$ is assumed to be small. For these small values of $\delta$, the magnetic field oscillates with frequency $\Omega_{0}$. This forcing term is assumed to be of $O\left(\varepsilon^{2}\right)$. Numerical diagnostics reveal the onset of chaos as a consequence.

The rest of this paper is organized as follows. Sec. 2 develops the asymptotic expansion for the basic equations and derives the governing generic problem at all orders of the perturbation expansion. Sec. 3 reviews and extends the results for the linear problem. The second-order equations are solved in Sec. 4. In particular, the second-order zerothharmonic yields the equations for the slow evolution of the equilibrium quantities on the second-order scales, while the second-harmonic equations yield the particular solutions at this order. Sec. 5 considers the third-order equation with the first harmonic yielding the overall equation for the evolution of the linear amplitude on the slow first-order scales. Sec. 6 discusses this amplitude equation to delineate the parameter regimes where the nonlinear evolution does or does not lead to a final stable or permanent wave structure or nonlinear pattern. In particular, the complex nonlinearity allows the existence of quasiperiodic and chaotic wave envelopes, unlike in earlier physical models governed by nonlinear Klein-Gordon equations. In addition, occurrence of chaos as a consequence of modulation of the external magnetic field is also considered. 
2. Basic Equations. The equations for a compressible, inviscid, infinitely conducting plasma are:

$$
\begin{gathered}
\rho \frac{d \vec{v}}{d t}=-\vec{\nabla} \cdot \vec{p}+\frac{1}{4 \pi}(\vec{\nabla} \times \vec{B}) \times \vec{B}, \\
\frac{\partial \rho}{\partial t}=-\vec{\nabla} \cdot(\rho \vec{v}), \\
\frac{\partial \vec{B}}{\partial t}=\vec{\nabla} \times(\vec{v} \times \vec{B}), \\
\vec{\nabla} \cdot \vec{B}=0, \\
\vec{p}=p_{\perp} \vec{I}+\left(p_{\|}-p_{\perp}\right) \hat{n} \hat{n}, \\
\vec{I}=\text { unit dyadic, }
\end{gathered}
$$

and the adiabatic equations of state with generalized polytrope exponents $\alpha, \beta, \epsilon$, and $\gamma$ are (Abraham-Shrauner, 1973):

$$
\frac{d}{d t}\left(\frac{p_{\|} B^{\alpha}}{\rho^{\gamma}}\right)=0
$$

and

$$
\frac{d}{d t}\left(\frac{p_{\perp}}{\rho^{\epsilon} B^{\beta}}\right)=0 .
$$

Here, $\|$ and $\perp$ denote components parallel and perpendicular to the magnetic field, respectively. The equilibrium we consider (Fig. 1) has a uniform magnetic field $\mathbf{B}=B_{0} \hat{z}$, constant density $\rho$, pressures $p_{\|}$and $p_{\perp}$, and a flow velocity $v_{0}(x)=v_{0 y}(x) \hat{y}+v_{0 z}(x) \hat{z}$, with a tangential velocity discontinuity at $x=0$.

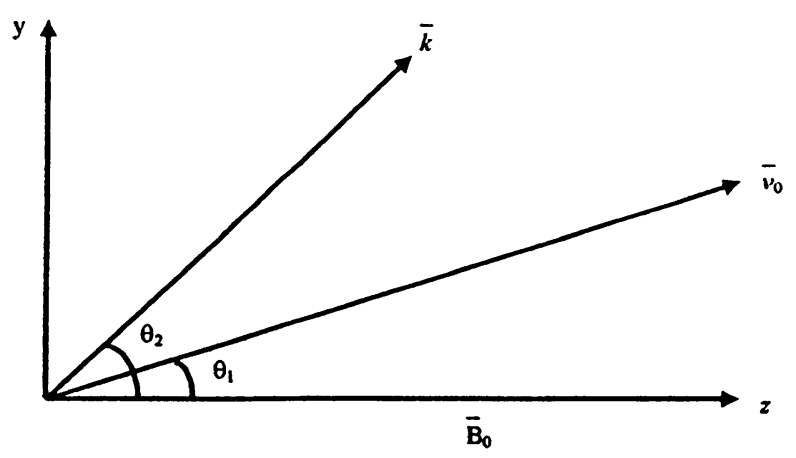

FIG. 1. Geometry of the equilibrium configuration and flow for the compressible tangential velocity discontinuity. The wave vector $\bar{k}$ for the perturbation quantities is shown. Perturbations $\pm \theta_{2}$ are degenerate

Each of the physical variables is expanded in a perturbation series

$$
\delta=\delta_{0}+\sum_{i=1} \varepsilon^{i} \delta^{(i)},
$$


where $\delta$ stands for $v_{x}, v_{y}, v_{z}, \rho, B_{x}, B_{y}, B_{z}, p_{\|}$, or $p_{\perp}$. As explained later, the expansion parameter $\varepsilon$ is a measure of the deviation from the extremum of the linear neutral curve. Introducing slow time and spatial scales $T_{i}, Y_{i}$, and $Z_{i}$ such that

$$
\begin{gathered}
T_{1}=\varepsilon t, \quad Y_{1}=\varepsilon y, \quad Z_{1}=\varepsilon z, \\
T_{2}=\varepsilon^{2} t, \quad Y_{2}=\varepsilon^{2} y, \quad Z_{2}=\varepsilon^{2} z,
\end{gathered}
$$

the temporal and spatial derivatives become

$$
\begin{aligned}
& \frac{\partial}{\partial t}=\frac{\partial}{\partial t}+\varepsilon \frac{\partial}{\partial T_{1}}+\varepsilon^{2} \frac{\partial}{\partial T_{2}} \\
& \frac{\partial}{\partial y}=\frac{\partial}{\partial y}+\varepsilon \frac{\partial}{\partial Y_{1}}+\varepsilon^{2} \frac{\partial}{\partial Y_{2}} \\
& \frac{\partial}{\partial z}=\frac{\partial}{\partial z}+\varepsilon \frac{\partial}{\partial Z_{1}}+\varepsilon^{2} \frac{\partial}{\partial Z_{2}}
\end{aligned}
$$

Using (2) and (3) in (1) yields equations at $O(\varepsilon), O\left(\varepsilon^{2}\right)$, and $O\left(\varepsilon^{3}\right)$. These equations and all subsequent expressions were verified using the computer algebra system Mathematica. The structure of these equations may be written as:

$$
\begin{gathered}
{\left[v_{0 y}(x) \frac{\partial}{\partial y}+v_{0 z}(x) \frac{\partial}{\partial z}+\frac{\partial}{\partial t}\right] \rho^{(i)}+\rho_{0}\left(\frac{\partial v_{x}^{(i)}}{\partial x}+\frac{\partial v_{y}^{(i)}}{\partial y}+\frac{\partial v_{z}^{(i)}}{\partial z}\right)=S_{1}^{(i)}} \\
\rho_{0}\left[v_{0 y}(x) \frac{\partial}{\partial y}+v_{0 z}(x) \frac{\partial}{\partial z}+\frac{\partial}{\partial t}\right] v_{z}^{(i)}+\frac{\partial p_{\|}^{(i)}}{\partial z}\left(\frac{p_{\perp 0}-p_{\| 0}}{B_{0}}\right) \frac{\partial B_{z}^{(i)}}{\partial z}=S_{2}^{(i)} \\
\rho_{0}\left[v_{0 y}(x) \frac{\partial}{\partial y}+v_{0 z}(x) \frac{\partial}{\partial z}+\frac{\partial}{\partial t}\right] v_{x}^{(i)}+\frac{\partial p_{\perp}^{(i)}}{\partial x}+\frac{B_{0}}{4 \pi} \frac{\partial B_{z}^{(i)}}{\partial x} \\
-\frac{B_{0}}{4 \pi}\left(\frac{p_{\perp 0}-p_{\| 0}}{B_{0}^{2} / 4 \pi}+1\right) \frac{\partial B_{x}^{(i)}}{\partial z}=S_{3}^{(i)} \\
\rho_{0}\left[v_{0 y}(x) \frac{\partial}{\partial y}+v_{0 z} \frac{\partial}{\partial z}+\frac{\partial}{\partial t}\right] v_{y}^{(i)}+\frac{\partial p_{\perp}^{(i)}}{\partial y}+\frac{B_{0}}{4 \pi} \frac{\partial B_{z}^{(i)}}{\partial y} \\
\frac{p_{\perp}^{(i)}}{p_{\perp 0}}-\frac{\beta B_{z}^{(i)}}{B_{0}}-\frac{\epsilon \rho^{(i)}}{\rho_{0}}=S_{5}^{(i)}\left(\frac{p_{\perp 0}-p_{\| 0}}{B_{0}^{2} / 4 \pi}+1\right) \frac{\partial B_{y}^{(i)}}{\partial z}=S_{4}^{(i)} \\
\frac{p_{\|}^{(i)}}{p_{\| 0}}+\frac{\alpha B_{z}^{(i)}}{B_{0}}-\frac{\gamma \rho^{(i)}}{\rho_{0}}=S_{6}^{(i)} \\
{\left[v_{0 y}(x) \frac{\partial}{\partial y}+v_{0 z}(x) \frac{\partial}{\partial z}+\frac{\partial}{\partial t}\right] B_{x}^{(i)}-B_{0} \frac{\partial v_{x}^{(i)}}{\partial z}=S_{7}^{(i)}} \\
\left.v_{0 y}(x) \frac{\partial}{\partial y}+v_{0 z}(x) \frac{\partial}{\partial z}+\frac{\partial}{\partial t}\right] B_{y}^{(i)}-B_{0} \frac{\partial v_{y}^{(i)}}{\partial z}-B_{x}^{(i)} \frac{\partial v_{0 y}(x)}{\partial x}=S_{8}^{(i)}
\end{gathered}
$$




$$
\begin{aligned}
& {\left[v_{0 y}(x) \frac{\partial}{\partial y}+v_{0 z} \frac{\partial}{\partial z}+\frac{\partial}{\partial t}\right] B_{z}^{(i)} }-B_{0} \frac{\partial v_{z}^{(i)}}{\partial z}-B_{x}^{(i)} \frac{\partial v_{0 z}(x)}{\partial x} \\
&+B_{0}\left[\frac{\partial v_{x}^{(i)}}{\partial x}+\frac{\partial v_{y}^{(i)}}{\partial y}+\frac{\partial v_{z}^{(i)}}{\partial z}\right]=S_{9}^{(i)} \\
& \frac{\partial B_{x}^{(i)}}{\partial x}+\frac{\partial B_{y}^{(i)}}{\partial y}+\frac{\partial B_{z}^{(i)}}{\partial z}=S_{10}^{(i)}
\end{aligned}
$$

for various source terms $S_{1}^{(i)}, S_{2}^{(i)}, S_{3}^{(i)}, \ldots, S_{10}^{(i)}$ where the superscript $(i)$ represents the power of $\varepsilon$, or the order of the term. Combining (4)-(13) will yield the generic structure of the underlying problem at linear, second, and third orders. For convenience, we define

$$
D \equiv\left[v_{0 y}(x) \frac{\partial}{\partial y}+v_{0 z}(x) \frac{\partial}{\partial z}+\frac{\partial}{\partial t}\right] .
$$

After combining Eqs. (4)-(13) we find that the composite generic form of the equation for the $x$ component of the magnetic field at all orders $i$ is (Brown and Roy Choudhury, 1998):

$$
\begin{aligned}
L B_{x}^{(i)}= & {\left[\frac{\rho_{0}}{B_{0}} D_{8} D_{1} D^{3}-\epsilon p_{\perp 0}\left(D_{8} D_{3}-D_{7} D_{2}\right) \frac{\partial^{2}}{\partial x \partial z}-\left(\frac{\beta p_{\perp 0}}{B_{0}}+\frac{B_{0}}{4 \pi}\right) D_{7} D_{1} \frac{\partial^{2}}{\partial x \partial z}\right.} \\
& \left.-\frac{B_{0}}{4 \pi}\left(\frac{p_{\perp 0}-p_{\| 0}}{B_{0}^{2} / 4 \pi}+1\right) D_{8} D_{1} D \frac{\partial^{2}}{\partial z^{2}}\right] B_{x}^{(i)}=\Gamma^{(i)},
\end{aligned}
$$

where the $i$-th order source term is given by

$$
\begin{aligned}
\Gamma^{(i)}= & \frac{\rho_{0}}{B_{0}} D_{8} D_{1} D^{2} S_{7}^{(i)}+\left[\epsilon p_{\perp 0} D_{4} D_{2}-\left(\frac{\beta p_{\perp 0}}{B_{0}}+\frac{B_{0}}{4 \pi}\right) D_{4} D_{1} D\right] \frac{\partial^{2} S_{10}^{(i)}}{\partial x \partial z} \\
& +D_{8} D_{1} D \frac{\partial S_{3}^{(i)}}{\partial z}+\left[\left(\frac{\beta p_{\perp 0}}{B_{0}}+\frac{B_{0}}{4 \pi}\right) D_{1} D-\epsilon p_{\perp 0} D_{2}\right] \frac{\partial^{3} \chi_{N 2}}{\partial x \partial y \partial z} \\
& -p_{\perp 0}\left(\frac{\epsilon}{\rho_{0}}+1\right) D_{8} D_{1} \frac{\partial^{2}\left\{S_{1}^{(i)}+S_{5}^{(i)}\right\}}{\partial x \partial z}+\epsilon p_{\perp 0} D_{8} \frac{\partial^{2} \chi_{N 1}}{\partial x \partial z} .
\end{aligned}
$$

Notice that the operator $L$ does not contain a constant term, i.e., one not containing a derivative.

3. The linear problem. Now we would like to briefly recapitulate the results for the $i=1$ or linear problem, which will be used in the higher-order calculations. Equations (4)-(13) for $i=1$ have source terms

$$
S_{1}^{(1)}=S_{2}^{(1)}=S_{3}^{(1)}=\cdots=S_{10}^{(1)}=0,
$$

which gives $\Gamma^{(1)} \equiv 0$. Also, the first order perturbation quantities are of the form

$$
\delta^{(1)}=f\left(x, Y_{1}, Y_{2}, Z_{1}, Z_{2}, T_{1}, T_{2}\right) \exp \left[i\left(k_{y} y+k_{z} z-\omega t\right)\right],
$$

with $\delta$ representing any of the field variables $v_{x}, v_{y}, v_{z}, \rho, B_{x}, B_{y}, B_{z}, p_{\|}$, or $p_{\perp}$. As a result, Eq. (14) gives $D=i\left(v_{0 y}(x) k_{y}+v_{0 z}(x) k_{z}-\omega\right) \equiv i \Omega$. Using this and Eq. (16), Eq. 
(15a) reduces to Eq. (3) of (Brown and Choudhury, 1998)

$$
\begin{aligned}
\frac{i \rho \Omega^{2} B_{x}^{(1)}}{k_{z} B_{0}} & +p_{\perp}\left[\frac{i \epsilon\left(\chi_{2} B_{x}^{(1)}+\chi_{3} B_{z}^{(1)}\right)}{\Omega}+\beta \frac{B_{z}^{(1)}}{B_{0}}\right]^{\prime}-\frac{B_{0}}{4 \pi}\left[\chi_{7}\left(B_{x}^{(1)^{\prime}}+i k_{y} \chi_{4} B_{x}^{(1)}\right)\right]^{\prime} \\
& -\frac{i k_{z} B_{0}}{4 \pi}\left(\frac{p_{\perp}-p_{\|}}{B_{0}^{2} / 4 \pi}+1\right) B_{x}^{(1)}=0,
\end{aligned}
$$

where $B_{z}^{(1)}$ is given by

$$
B_{z}^{(1)}=-\chi_{7}\left(B_{x}^{(1)^{\prime}}+i k_{y} \chi_{4} B_{x}^{(1)}\right) .
$$

Equation (17a) is solved with boundary conditions for $x \rightarrow \pm \infty$, together with integrating across the jump at $x=0$ between the two half-spaces $x<0$ and $x>0$. We consider the conditions at $x \rightarrow \pm \infty$ first. Away from the discontinuity at $x=0$, the solutions of $(17 \mathrm{a})$ are $B_{x}^{(1)}=$ constant $\exp \left( \pm i k_{-} x\right)$ for $x<0$, and $B_{x}^{(1)}=$ constant $\exp \left( \pm i k_{+} x\right)$ for $x>0$, where

$$
k_{ \pm}^{2}=\frac{k_{z}^{2}\left[\left(u_{D \pm}^{2}-\gamma r^{2}\right)\left\{u_{D \pm}^{2}-\left(1-r^{2}+q_{D}^{2}\right)\right\}-T^{2}\left\{\left(\beta+q_{D}^{2}\right)\left(u_{D \pm}^{2}-\gamma r^{2}\right)-\epsilon\left((1+\alpha) r^{2}-1-u_{D \pm}^{2}\right)\right\}\right\}}{\left(\beta+\epsilon+q_{D}^{2}\right)\left(u_{D \pm}^{2}-\gamma r^{2}\right)+\epsilon\left\{(\gamma-1-\alpha) r^{2}+1\right\}} .
$$

Here, we have introduced the dimensionless frequency

$$
W_{D} \equiv \frac{\omega}{k_{z} S_{\perp}}=W_{r}+i W_{i},
$$

and the dimensionless flow velocity

$$
u_{D}=\frac{\Omega}{k_{z} S_{\perp}}= \pm \frac{M F}{2}-W_{D}
$$

where $S_{\|, \perp} \equiv\left(\frac{p_{\|, \perp}}{\rho}\right)^{\frac{1}{2}}$ are the sound speeds parallel and perpendicular to the magnetic field. We also define the dimensionless anisotropy parameter

$$
r=\frac{S_{\|}}{S_{\perp}}
$$

and the ratio of the magnetic field-energy density to the perpendicular thermal energy density (the inverse plasma beta)

$$
q_{D}^{2} \equiv\left(\frac{v_{A}}{S_{\perp}}\right)^{2}
$$

where the Alfvén speed is

$$
v_{A}^{2} \equiv \frac{B_{0}^{2}}{4 \pi \rho} .
$$

The correct choice of the \pm signs in the external regions is determined by dual considerations. The $x$ component of the magnetic field perturbation $B_{x}^{(1)}$ should correspond to a spatially damping (in $x$ ), and outgoing mode at $x \rightarrow \pm \infty$ in the comoving frame of the plasma in each of the external regions (Roy Choudhury and Lovelace 1984 \& 1986; Miura and Pritchett 1982). The requirement of damping follows since there is no source of energy away from $x=0$. If we choose the solutions $B_{x}^{(1)}=\operatorname{constant} \exp \left(-i k_{-} x\right)$ for $x<0$, and $B_{x}^{(1)}=$ constant $\exp \left(-i k_{+} x\right)$ for $x>0$, this results in outgoing, spatially damping modes for $x \rightarrow \pm \infty$. Combining these with (16) and (18) yields

$$
B_{x}^{(1)}=A\left(Y_{1}, Y_{2}, Z_{1}, Z_{2}, T_{1}, T_{2}\right) e^{i \theta}+\text { c.c. }, \quad x \neq 0
$$


with

$$
\theta=k_{y} y+k_{z} z-\omega t-k_{ \pm} x .
$$

$A$ is the amplitude of the $x$ component of the magnetic field perturbation and c.c. denotes complex conjugate. Using $(20)$, the other $O(\varepsilon)$ equations yield the solutions for the other linear fields (away from $x=0$ ). Integrating (17a) across the jump at $x=0$, and denoting quantities for $x>0$ and $x<0$ respectively by subscripts + and - , we may obtain the linear dispersion relation. This has been considered in detail by Brown and Choudhury (1998) to map out the regions of linear instability.

Here we give two examples of regions of instability for later use in the nonlinear analysis. First we consider the CGL (Chew-Goldberger-Low) case, for which the polytrope indices are $\epsilon=\beta=1, \alpha=2$, and $\gamma=3$. Fig. 2 shows the unstable standing wave regimes for $r=0.1$ and $T=10\left(\theta_{2}=84.29^{\circ}\right)$. The curve here is given by $q_{D}=\left(r^{2}-1+\frac{M^{2} F^{2}}{4}\right)^{\frac{1}{2}}$ (Brown and Choudhury, 1998). There is a lower limiting curve for the regime of instability but it is not present for $M F<40$.

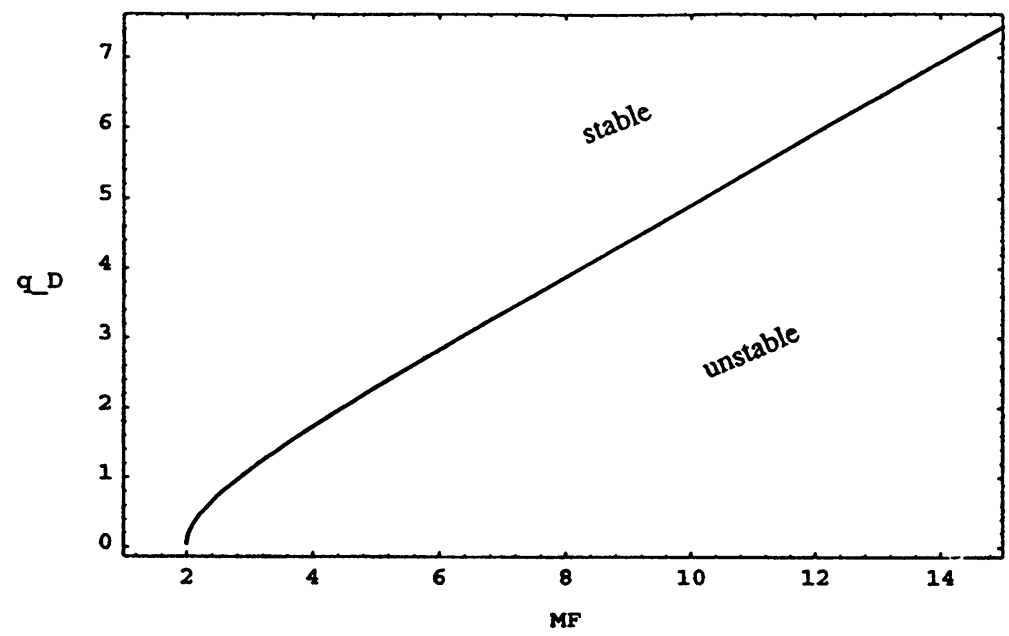

FIG. 2. The unstable standing wave regimes in the $\left(M F, q_{D}\right)$ plane for $r=0.1$ and $T=10\left(\theta_{2}=84.29^{\circ}\right)$, for the CGL wave case (polytrope indices $\epsilon=\beta=1, \alpha=2$, and $\gamma=3$ ). In the intermediate region, the vortex sheet is unstable and the growth rate is given in (Brown and Choudhury, 1998). The limiting curve is given by $q_{D}=\left(r^{2}-1+\frac{M^{2} F^{2}}{4}\right)^{\frac{1}{2}}$

Next, we give an example from the MHD (Magnetohydrodynamic) case, for which the polytrope indices are $\alpha=0, \beta=0, \epsilon=\frac{5}{3}, \gamma=\frac{5}{3}$. For the collision-dominated MHD case, the pressure is isotropic and $r=1$. Fig. 3 shows the unstable standing wave regimes for $T=10\left(\theta_{2}=84.29^{\circ}\right)$.

4. Second-order solutions. To consider the nonlinear evolution within the usual weakly nonlinear theory we consider the Stuart-type expansion which is of the form 


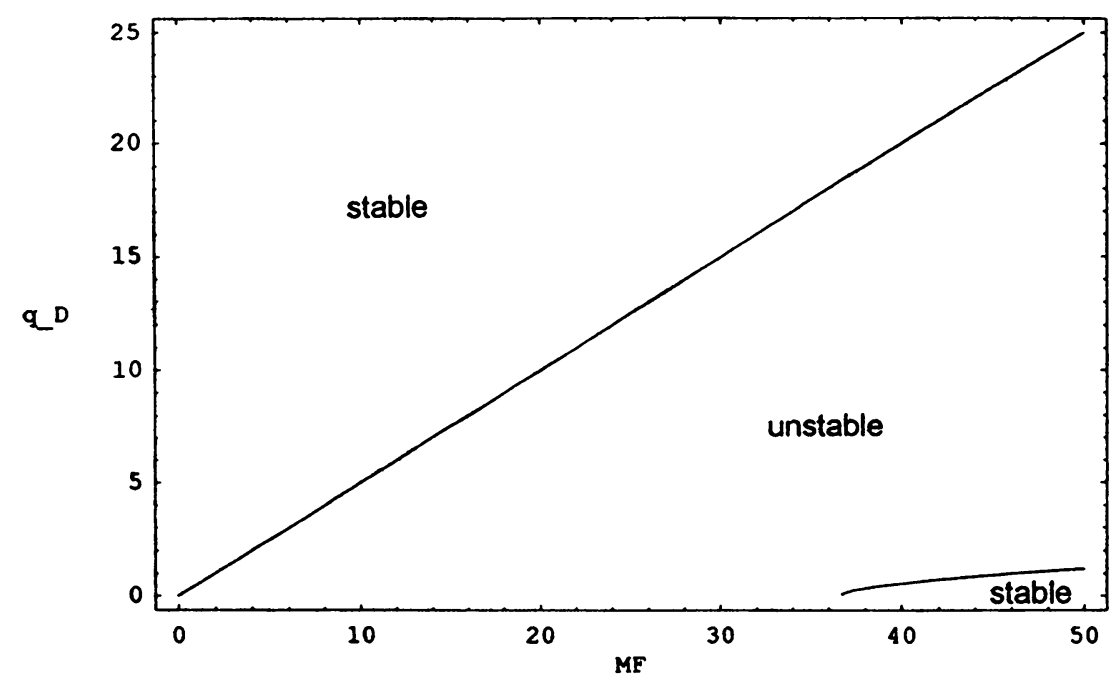

FIG. 3. The unstable standing wave regimes in the $\left(M F, q_{D}\right)$ plane for $r=1$ and $T=10\left(\theta_{2}=84.29^{\circ}\right)$, for the MHD wave case (polytrope indices $\alpha=\beta=0, \epsilon=\gamma=\frac{5}{3}$ ). In the intermediate region, the vortex sheet is unstable and the growth rate is given in (Brown and Choudhury, 1998)

(Dodd et al., 1982; Drazin and Reid, 1981; Gibbon and McGuinness, 1981; Stuart, 1960)

$$
M F=M F_{c} \pm \varepsilon^{2} \mu_{2}
$$

in the case of a most unstable wavenumber $k_{c}$, which first goes unstable at $M F=M F_{c}$. However, here $M F$ is independent of $k$ since, in the linear dispersion relation, $k$ is balanced on both sides and when solving for $M F, k$ cancels out. In this case, the most unstable mode occurs at $\theta_{2}=\frac{\pi}{2}$ since at this angle the stabilizing effect of the magnetic field is minimum. Picking $\theta_{2}$ large will give the most unstable Mach number at any angle $\theta_{1}$ of $v_{0}$ to $\mathbf{B}_{0}$.

The $O\left(\varepsilon^{2}\right)$ source terms $S_{1}^{(2)}, S_{2}^{(2)}, S_{3}^{(2)}, \ldots, S_{10}^{(2)}$ in the two half-spaces are computed using (20), (21) and the solutions for the other linear fields. Each of these terms has the structure

$$
\delta^{(2)}=\delta_{0}^{(2)}+\delta_{1}^{(2)} e^{i \theta}+\delta_{2}^{(2)} e^{2 i \theta},
$$

where $\delta$ stands for $S_{1}^{(2)}, S_{2}^{(2)}, S_{3}^{(2)}, \ldots$, or $S_{10}^{(2)}$. Using these, the inhomogeneous term on the right-hand side of (15a) may be computed, yielding the equation

$$
L B_{x}^{(2)}=\Gamma_{0}^{(2)}+\Gamma_{1}^{(2)} e^{i \theta}+\Gamma_{2}^{(2)} e^{2 i \theta}+\text { c.c. },
$$

with $\Gamma_{0}^{(2)}, \Gamma_{1}^{(2)}, \Gamma_{2}^{(2)}$ given by (15b) with $i=2$ and $\delta^{(2)}$ of $(22)$.

The zeroth-harmonic term $\Gamma_{0}^{(2)}$ in $(23)$ is secular and would cause $B_{x}^{(2)}$ to contain terms proportional to $x$ and $t$, causing expansions (2) to become non-uniform after a time of $O\left(\varepsilon^{-1}\right)$ since the operator $L$ does not contain a constant term (Dodd et al., 1982; Nayfeh, 1973), as noted following (15). To suppress this secular term, one would need to set $\Gamma_{0}^{(2)}=0$. We will not pursue this here since it will shortly be demonstrated that 
one needs to look at the individual zeroth-harmonic source terms $S_{1,0}^{(2)}, S_{2,0}^{(2)}, S_{3,0}^{(2)}, \ldots$, and $S_{10,0}^{(2)}$ in (22) (to suppress such secular terms), which takes the above condition $\Gamma_{0}^{(2)}=0$ into account automatically.

The first-harmonic term $\Gamma_{1}^{(2)} e^{i \theta}$ in (23) is also secular (since the solutions of the homogeneous equation have the form $\left.e^{i \theta}\right)$. Suppressing this requires $\Gamma_{1}^{(2)}=0$.

With the secular terms $\Gamma_{0}^{(2)}$ and $\Gamma_{1}^{(2)}$ suppressed in (23), a particular solution of (23) for the second-order $x$ component of the magnetic field

$$
B_{x}^{(2)}=B_{x, 0}^{(2)}\left(Y_{1}, Y_{2}, Z_{1}, Z_{2}, T_{1}, T_{2}\right)+\left(c A^{2} e^{2 i \theta}+\text { c.c. }\right)
$$

with

$$
\begin{aligned}
& c=\frac{\Gamma_{2}^{(2)}}{-2 i \rho_{0} H^{4}\left\{4 k_{y}^{2} k_{z} \rho_{0}^{2}\left[\beta+\epsilon+\frac{B_{0}^{3}}{4 \pi}\right] H^{4}+4 k_{z}^{3} \rho_{0}^{2} B_{0}\left[(2 \gamma-1) p_{\| 0}+p_{\perp 0}+\frac{B_{0}^{2}}{4 \pi}\right] H^{4}\right.} \\
& +16 k_{y}^{2} k_{z}^{3}\left[2 \gamma \rho_{0} p_{\| 0}\left(\beta+\frac{B_{0}^{3}}{4 \pi}\right)+\frac{\epsilon}{B_{0}}\left(\left(1-\alpha+\gamma \rho_{0} B_{0}\right) p_{\| 0}-p_{\perp 0}\right)\right] H^{2} \\
& +16 k_{z}^{5} \rho_{0} B_{0} p_{\| 0} \gamma\left[2\left(p_{\perp 0}-p_{\| 0}+\frac{B_{0}^{2}}{4 \pi}\right)+\gamma p_{\| 0}\right] H^{2}+k_{z} \rho_{0}^{3} B_{0} H^{6} \\
& +64 k_{y}^{2} k_{z}^{5} p_{\| 0} \gamma\left[p_{\| 0} \gamma\left(\beta+\frac{B_{0}^{3}}{4 \pi}\right)+\epsilon\left((1-\alpha) p_{\| 0}-p_{\perp 0}\right)\right] \\
& \left.+64 k_{z}^{7} B_{0} p_{\| 0}^{2}\left(p_{\perp 0}-p_{\| 0}+\frac{B_{0}^{2}}{4 \pi}\right)\right\} \\
& -\left(\beta B_{0} p_{\perp 0}+\frac{B_{0}^{3}}{4 \pi}\right) 8 i k_{z} k_{ \pm}^{2} H^{2}\left\{4 k_{z}^{2} \rho_{0}^{2}\left[(2 \gamma-1) p_{\| 0}+p_{\perp 0}+\frac{B_{0}^{2}}{4 \pi}\right] H^{4}+\rho_{0}^{3} H^{6}\right. \\
& -32 k_{z}^{4} \rho_{0} p_{\| 0} \gamma\left[\left(\frac{\gamma}{2}-1\right) p_{\| 0}+p_{\perp 0}+\frac{B_{0}^{2}}{4 \pi}\right] H^{2} \\
& \left.+64 k_{z}^{6} p_{\| 0}^{2} \gamma^{2}\left(p_{\perp 0}-p_{\| 0}+\frac{B_{0}^{2}}{4 \pi}\right)\right\} \\
& -8 i k_{z} k_{ \pm}^{2} p_{\perp 0} \epsilon B_{0} H^{2}\left\{16 k_{z}^{4} \rho_{0}\left[\gamma p_{\| 0} \chi_{1}+\chi_{2}\left(p_{\perp 0}-p_{\| 0}+\frac{B_{0}^{2}}{4 \pi}\right)\right] H^{2}\right. \\
& +64 k_{z}^{6} p_{\| 0} \gamma \chi_{1}\left(p_{\perp 0}-p_{\| 0}+\frac{B_{0}^{2}}{4 \pi}\right)+4 k_{z}^{2} \rho_{0}^{2}\left[(\gamma-\alpha) p_{\| 0}+\frac{B_{0}^{2}}{4 \pi}\right] H^{4} \\
& \left.+\rho_{0}^{3} H^{6}\right\} \\
& -8 i k_{z}^{3}\left(p_{\perp 0}-p_{\| 0}+\frac{B_{0}^{2}}{4 \pi}\right) H^{2}\left\{4 k_{y}^{2} \rho_{0}^{2}\left[\beta+\epsilon+\frac{B_{0}^{3}}{4 \pi}\right] H^{4}\right. \\
& +4 k_{z}^{2} \rho_{0}^{2} B_{0}\left[(2 \gamma-1) p_{\| 0}+p_{\perp 0}+\frac{B_{0}^{2}}{4 \pi}\right] H^{4}
\end{aligned}
$$




$$
\begin{aligned}
& +16 k_{y}^{2} k_{z}^{2}\left[2 \gamma \rho_{0} p_{\| 0}\left(\beta+\frac{B_{0}^{3}}{4 \pi}\right)+\frac{\epsilon}{B_{0}}\left(\left(1-\alpha+\gamma \rho_{0} B_{0}\right) p_{\| 0}-p_{\perp 0}\right)\right] H^{2} \\
& +16 k_{z}^{4} \rho_{0} B_{0} p_{\| 0} \gamma\left[2\left(p_{\perp 0}-p_{\| 0}+\frac{B_{0}^{2}}{4 \pi}\right)+\gamma p_{\| 0}\right] H^{2}+\rho_{0}^{3} B_{0} H^{6} \\
& +64 k_{y}^{2} k_{z}^{4} p_{\| 0} \gamma\left[p_{\| 0} \gamma\left(\beta+\frac{B_{0}^{3}}{4 \pi}\right)+\epsilon\left((1-\alpha) p_{\| 0}-p_{\perp 0}\right)\right] \\
& \left.+64 k_{z}^{6} p_{\| 0}^{2} B_{0} \gamma\left(p_{\perp 0}-p_{\| 0}+\frac{B_{0}^{2}}{4 \pi}\right)\right\}
\end{aligned}
$$

where

$$
H=2\left(v_{0 y}(x) k_{y}+v_{0 z}(x) k_{z}-\omega\right) .
$$

Returning to the individual second-order equations, and writing the individual secondorder fields in the form

$$
\phi^{(2)}=\phi_{0}^{(2)}+\phi_{1}^{(2)} e^{i \theta}+\phi_{2}^{(2)} e^{2 i \theta}+\text { c.c. }
$$

with $\phi$ representing $v_{x}, v_{y}, v_{z}, \rho, B_{x}, B_{y}, B_{z}, p_{\|}$, or $p_{\perp}$, a typical equation yields

$$
\begin{aligned}
\frac{\partial v_{x}^{(2)}}{\partial z} & =i k_{z} v_{x, 1}^{(2)} e^{i \theta}+2 i k_{z} v_{x, 2}^{(2)} e^{2 i \theta} \\
& =\frac{1}{B_{0}}\left[2 i\left(v_{0 y}(x) k_{y}+v_{0 z}(x) k_{z}-\omega\right) c A^{2} e^{2 i \theta}-S_{7,0}^{(2)}-S_{7,1}^{(2)} e^{i \theta}-S_{7,2}^{(2)} e^{2 i \theta}\right]
\end{aligned}
$$

Hence,

$$
S_{7,0}^{(2)}=0
$$

(Alternatively, balancing $S_{7,0}^{(2)}$ would require secular terms proportional to $t$ and $z$ in $v_{x}^{(2)}$. Note that such terms are inadmissible since we work with physical field variables and the boundary conditions at $x \rightarrow+\infty$ are violated by such terms.) Similar considerations imply

$$
S_{j, 0}^{(2)}=0, \quad j=1,2,3, \ldots, 10 .
$$

Using (28), $\Gamma_{0}^{(2)}$ (from $\left.(15 \mathrm{~b})\right) \equiv 0$, so the zeroth-harmonic secular term in (23) is indeed zero, as required earlier. The suppression of second-order zeroth-harmonic terms represented by (28) are conditions for the evolution of the equilibrium quantities $B_{0}, p_{\| 0}, p_{\perp 0}, \rho_{0}$, and $v_{0}(x)$ on the even slower second-order scales $Y_{2}, Z_{2}$, and $T_{2}$.

5. Third-order analysis and amplitude evolution equations. Comparing (4)(13) for $i=3$ with the $O\left(\varepsilon^{3}\right)$ sources yields $S_{1}^{(3)}, S_{2}^{(3)}, S_{3}^{(3)}, \ldots$, and $S_{10}^{(3)}$. Computing these using (20), (21), the solutions for the other linear fields, (24), and (26) (with $\phi$ standing for $v_{x}, v_{y}, v_{z}, \rho, B_{x}, B_{y}, B_{z}, p_{\|}$, or $\left.p_{\perp}\right)$ yields

$$
\delta^{(3)}=\delta_{0}^{(3)}+\delta_{1}^{(3)} e^{i \theta}+\delta_{2}^{(3)} e^{2 i \theta}+\delta_{3}^{(3)} e^{3 i \theta}+\text { c.c. },
$$

where $\delta$ stands for $S_{1}^{(3)}, S_{2}^{(3)}, S_{3}^{(3)}, \ldots$, or $S_{10}^{(3)}$. Note that we do not use the linear and second-order solutions to explicitly expand these coefficients for $i=2,3$ as they will not 
be required in our analysis. Once these coefficients are known, the right-hand side of (15a) may be evaluated for $i=3$, yielding the equation

$$
L B_{x}^{(3)}=\Gamma_{0}^{(3)}+\Gamma_{1}^{(3)} e^{i \theta}+\Gamma_{2}^{(3)} e^{2 i \theta}+\Gamma_{3}^{(3)} e^{3 i \theta}+\text { c.c. },
$$

with $\Gamma_{i}^{(3)}, i=0,1$, given in terms of these by (15b) with $i=3$.

(a) Zeroth harmonic

Considering the structure of the third-order equations, the operator $D \equiv\left(v_{0 y}(x) \frac{\partial}{\partial y}+\right.$ $\left.v_{0 z}(x) \frac{\partial}{\partial z}+\frac{\partial}{\partial t}\right)$ has no constant term. Hence, as in second-order, the third-order zerothharmonic sources must vanish, i.e.,

$$
S_{j, 0}^{(3)}=0, \quad j=1,2,3, \ldots, 10
$$

to prevent the occurrence of aperiodic $z$ or $t$ dependent terms in the third-order zerothharmonic fields $v_{x, 0}^{(3)}, v_{y, 0}^{(3)}, v_{z, 0}^{(3)}, \rho_{0}^{(3)}, B_{x, 0}^{(3)}, B_{y, 0}^{(3)}, B_{z, 0}^{(3)}, p_{\|, 0}^{(3)}$, or $p_{\perp, 0}^{(3)}$ (such terms violate the boundary conditions on the fields and are inadmissible).

(b) First harmonic terms and evolution equation

The $\Gamma_{1}^{(3)} e^{i \theta}$ term on the right-hand side of (30) resonates with the homogeneous solution of this equation. Suppressing this term in the usual way so as to prevent the occurrence of non-uniform secular terms yields after considerable algebra using the symbolic computing system Mathematica,

$$
\begin{aligned}
\Gamma_{1}^{(3)}= & \chi_{103} A+\chi_{104}|A|^{2} A+\chi_{105} \frac{\partial^{2} A}{\partial Y_{1}^{2}}+\chi_{106} \frac{\partial^{2} A}{\partial Z_{1}^{2}}+\chi_{107} \frac{\partial^{2} A}{\partial T_{1}^{2}} \\
& +\chi_{108} \frac{\partial^{2} A}{\partial Y_{1} \partial Z_{1}}+\chi_{109} \frac{\partial^{2} A}{\partial Y_{1} \partial T_{1}}+\chi_{110} \frac{\partial^{2} A}{\partial Z_{1} \partial T_{1}} \\
& +\chi_{111} \frac{\partial A}{\partial T_{2}}+\chi_{112} \frac{\partial A}{\partial Y_{1}}+\chi_{115} \frac{\partial A}{\partial Y_{2}}+\chi_{116} \frac{\partial A}{\partial Z_{2}}=0,
\end{aligned}
$$

where the $\chi$ 's are complicated constants.

6. Discussion. The evolution equation (32) governing the weakly nonlinear evolution of the amplitude $A$ (of the linear fields of (19)-(21)) for the supersonic Kelvin-Helmholtz instability is of the form of a non-integrable nonlinear Klein-Gordon equation as for the incompressible Kelvin-Helmholtz instability (Weissman, 1979). In both these cases, we do not obtain the integrable $A B$ equations, which are the other possible form of the canonical evolution equation for inviscid or dispersive instabilities as discussed in Sec. 1 (Gibbon and McGuinness, 1981; Gibbon et al., 1979).

For linear physical or field variables of the form

$$
\xi^{(1)}=A\left(Y_{1}, Y_{2}, Z_{1}, Z_{2}, T_{1}, T_{2}\right) e^{i\left(k_{y} y+k_{z} z-\omega t\right)}
$$

satisfying a general linear dispersion

$$
F\left(\omega, k_{y}, k_{z}, v_{z m}\right)=0
$$


it may be shown (Dodd et al., 1982) that the general form of the nonlinear Klein-Gordon equation is

$$
\begin{gathered}
i\left(-F_{\omega} \frac{\partial A}{\partial T_{2}}+F_{k_{y}} \frac{\partial A}{\partial Y_{2}}+F_{k_{z}} \frac{\partial A}{\partial Z_{2}}\right)+\frac{1}{2} F_{\omega \omega} \frac{\partial^{2} A}{\partial T_{1}^{2}}-F_{\omega k_{y}} \frac{\partial^{2} A}{\partial T_{1} \partial Y_{1}} \\
+\frac{1}{2} F_{k_{y} k_{y}} \frac{\partial^{2} A}{\partial Y_{1}^{2}}+F_{k_{y} k_{z}} \frac{\partial^{2} A}{\partial Y_{1} \partial Z_{1}}+\frac{1}{2} F_{k_{z} k_{z}} \frac{\partial^{2} A}{\partial Z_{1}^{2}}-F_{\omega k_{z}} \frac{\partial^{2} A}{\partial T_{1} \partial Z_{1}} \\
=\alpha_{1} A+\alpha_{2}|A|^{2} A
\end{gathered}
$$

where

$$
-F_{\omega} \frac{\partial A}{\partial T_{1}}+F_{k_{y}} \frac{\partial A}{\partial Y_{1}}+F_{k_{z}} \frac{\partial A}{\partial Z_{1}}=0 .
$$

Note that our nonlinear evolution equation (32) is of this form.

In general, one may consider the general nonlinear evolution equation in one of three regions. The first is the linearly stable region where $F_{\omega}, F_{k_{y}}, F_{k_{z}} \neq 0$. The second region is on the neutral surface (or stability boundary) $\operatorname{Im} \omega=0$, but away from its extremum, so that $F_{\omega}=0$, but $F_{k_{y}}, F_{k_{z}} \neq 0$. And finally, there is the third region near the extremum of the neutral surface where $F_{\omega}=F_{k_{y}}=F_{k_{z}}=0$. In the first region, waves propagate as dispersive modes, yielding an evolution equation first derived by Benney and Newell (1967), and also considered by Nayfeh and Saric (1972), Benney and Roskes (1969), Hasimoto and Ono (1972), Davey and Stewartson (1974), and Weissman (1979). Since the linearly stable region is not our primary focus, we shall not consider this further. The second regime cannot be usefully treated within the framework of the weakly nonlinear analysis because, away from the extremum of the neutral surface, at any Mach number there are rapidly growing modes within the unstable region which make the perturbation expansions non-uniform. Hence, we shall concentrate on the third region.

Following Weissman (1979), we may recast Eq. (37) into the form

$$
\begin{gathered}
\left(\frac{\partial}{\partial T_{1}}+\sigma_{k_{z}}^{+} \frac{\partial}{\partial Y_{1}}\right) \\
\left(\frac{\partial}{\partial T_{1}}+\sigma_{k_{z}}^{-} \frac{\partial}{\partial Y_{1}}\right) A+\sigma_{k_{y}}^{+} \sigma_{k_{y}}^{-} \frac{\partial^{2} A}{\partial Z_{1}^{2}} \\
=G A+\bar{N}|A|^{2} A,
\end{gathered}
$$

where

$$
G \equiv \frac{2 \alpha_{1}}{F_{\omega \omega}}, \quad \bar{N} \equiv \frac{2 \alpha_{2}}{F_{\omega \omega}} .
$$

Also, $\sigma_{k_{y}}^{ \pm}$and $\sigma_{k_{z}}^{ \pm}$are the two values of the $y$ and $z$ group velocities $\sigma_{k_{y}}=-\frac{F_{k_{y}}}{F_{\omega}}$ and $\sigma_{k_{z}}=-\frac{F_{k_{z}}}{F_{\omega}}$, which may be expressed near the extremum of the neutral surface as

$$
\begin{gathered}
\sigma_{k_{z}}^{ \pm}=\frac{-F_{\omega k_{z}} \pm\left(F_{\omega k_{z}}^{2}-F_{\omega \omega} F_{k_{z} k_{z}}\right)^{\frac{1}{2}}}{F_{\omega \omega}}, \\
\sigma_{k_{y}}^{ \pm}= \pm\left(-\frac{F_{k_{y} k_{y}}}{F_{\omega \omega}}\right)^{\frac{1}{2}} .
\end{gathered}
$$

Defining a variable $Z^{\prime}=Z_{1}-c T_{1}$ in a frame moving with speed $c$, (37) takes the form

$$
\frac{\partial^{2} A}{\partial T_{1}^{2}}-\omega_{k_{z}}^{2} \frac{\partial^{2} A}{\partial Z^{\prime 2}}-\omega_{k_{y}}^{2} \frac{\partial^{2} A}{\partial Y_{1}^{2}}=G A+\bar{N}|A|^{2} A,
$$

which is a nonlinear Klein-Gordon equation. 
The nonlinear evolution equation $((37)$ or $(40))$ has various kinds of solutions. In particular, we may consider (Weissman, 1979; Malik and Singh, 1984 and references therein): (a) uniform, time-independent wave trains, (b) steady-state, space-dependent wave trains, (c) nonlinear envelopes or traveling wave solutions, and (d) modulational stability, or linearized stability of uniform solutions. These solutions have been extensively discussed by Weissman (1979), which also reviews the results of earlier treatments of this equation (Pedlosky, 1970; Drazin, 1970; Nayfeh and Saric, 1972). The effect of different initial conditions is also considered there. The modulational instability has been considered using numerical Floquet analysis by Murakami (1986) and Parkes (1991) both for uniform solutions $A=A_{0} e^{i \sigma t}$ ( $A_{0}$ real) corresponding to a wave train, as well as spatially uniform but temporally periodic Jacobian elliptic function solutions. Here, we shall primarily consider solutions in categories (a) and (c) above, referring the reader to the cited sources for other types of solutions and analysis. We shall find, however, that the dynamics of the solutions are very different from earlier cases due to our nonlinear coefficient $\bar{N}$ being complex. To the best of our knowledge, this has not been considered earlier. In addition, we shall also consider the occurrence of chaos due to the external modulation of the magnetic field.

(a) Uniform, time-dependent solutions

For the purpose of comparison, we shall first summarize the earlier solutions with $\bar{N}$ real. For a spatially uniform $A \equiv R e^{i \theta}$, the evolution equation ((37) or (40)) yields

$$
\begin{gathered}
\frac{d \theta}{d T_{1}}=\frac{\lambda}{R^{2}} \\
\frac{d^{2} R}{d T_{1}^{2}}=\frac{\lambda^{2}}{R^{3}}+G R+\bar{N} R^{3},
\end{gathered}
$$

where $\lambda=R(0)^{2} \frac{d \theta}{d T_{1}}(0)$ is an initial condition-dependent constant. Considering the simplest initial condition, that of constant phase $\frac{d \theta}{d T_{1}}=0$ at $T_{1}=0$ (following Nayfeh and Saric's treatment for a nonlinear Schrödinger equation (1972)) yields $\lambda=0$ and $\theta=\theta(0)=$ constant. For this case, one may use $A$ real and, via a phase-plane analysis (Nayfeh and Saric, 1972; Weissman, 1979) identify different possibilities for the nonlinear evolution based on the signs of $G$ and $\bar{N}$. These are:

a) $G<0, \bar{N}<0$. Both linear and nonlinear terms are stabilizing, and all solutions have the form of the elliptic function $c n$ and are bounded.

b) $G>0, \bar{N}<0$. The equation is linearly unstable $(G>0)$, but the nonlinear term is stabilizing yielding bounded oscillating solutions given by the $c n$ function outside the separatrix and the $d n$ function inside it.

c) $G<0, \bar{N}>0$. This case gives "nonlinear" or "subcritical" instability with instability for small amplitudes and solutions blowing up in a finite time if the initial amplitude is above a certain threshold amplitude.

d) $G>0, \bar{N}>0$. In this case, both the linear and nonlinear terms are destabilizing leading to super-exponential growth (so that the original perturbation expansions become non-uniform or break down after some point).

For numerical illustrations, we shall pick the following weakly nonlinear cases:

1. $M F=6, q_{D}=2.7, k_{y}=0.5, k_{z}=0.05$ (from Fig. 2); 
2. $M F=2.4, q_{D}=0.5, k_{y}=0.5, k_{z}=0.05$ (from Fig. 2);

3. $M F=2, q_{D}=0.9, k_{y}=0.5, k_{z}=0.05$ (from Fig. 3);

4. $M F=9, q_{D}=4, k_{y}=0.5, k_{z}=0.05$ (from Fig. 3).

For these cases, the various coefficients in the nonlinear evolution equation (35) are given in Table 1, where $\mu_{2}$ is the deviation from the critical value of $M F$ in the neutral surface in each case, and $\delta$ is the amplitude of the slow time-dependent modulation of the equilibrium magnetic field. Using these, and equation (38), the coefficients $G \equiv$ $G_{0}+\beta_{1} \cos \left(\Omega_{0} T_{1}\right)+\beta_{2} \sin \left(\Omega_{0} T_{1}\right)$ and $\bar{N}$ for the above four cases are:

1. $G_{0}=-0.0000218575 \mu_{2}$,

$\beta_{1}=0.0183596 \delta, \beta_{2}=-0.000499426 i \delta \Omega_{0}$,

$\bar{N} \equiv \bar{N}_{r}+i \bar{N}_{i}=0.00257845-0.00125968 i$.

2. $G_{0}=-0.0000437557 \mu_{2}$,

$\beta_{1}=0.0129796 \delta, \beta_{2}=-0.00010267 i \delta \Omega_{0}$,

$\bar{N} \equiv \bar{N}_{r}+i \bar{N}_{i}=-0.0559688-0.00138016 i$.

3. $G_{0}=9.65039 \times 10^{-7} \mu_{2}$,

$\beta_{1}=0.00128441 \delta, \beta_{2}=-4.32123 \times 10^{-6} i \delta \Omega_{0}$,

$\bar{N} \equiv \bar{N}_{r}+i \bar{N}_{i}=0.000402929-0.0000199259 i$.

$4 G_{0}=-0.0000365345 \mu_{2}$,

$\beta_{1}=0.0412971 \delta, \beta_{2}=-0.00281915 i \delta \Omega_{0}$,

$\bar{N} \equiv \bar{N}_{r}+i \bar{N}_{i}=0.00822425-0.0149168 i$.

In particular, we note that, as mentioned earlier, the coefficient $\bar{N}$ is complex. Note also that for each case we may use the entries in Table 1 to work out the group velocities $\sigma_{k_{y}}^{ \pm}$and $\sigma_{k_{z}}^{ \pm}$in $(39)$.

The complex value of $\bar{N}$ obtained here alters the dynamics of spatially uniform and spatially non-uniform solutions of (37) or (40) dramatically. An analogue is the difference between the real and complex Lorenz equations (Dodd et al., 1982). In particular, for spatially uniform $A \equiv R e^{i \theta}$, we now obtain

$$
\begin{gathered}
R \frac{d^{2} \theta}{d T_{1}^{2}}+2 \frac{d R}{d T_{1}} \frac{d \theta}{d T_{1}}-\bar{N}_{i} R^{3}=0 \\
\frac{d^{2} R}{d T_{1}^{2}}=R\left(\frac{d \theta}{d T_{1}}\right)^{2}+G_{0} R+\bar{N}_{r} R^{3}
\end{gathered}
$$


TABLE 1. The various coefficients in the nonlinear evolution equation (7.58) ( $\mu_{2}$ is the deviation from the critical value of $M F$ in the neutral surface, and $\delta$ is the amplitude of the slow time-dependent modulation of the equilibrium magnetic field)

\begin{tabular}{|c|ll|}
\hline & $F_{\omega \omega}=-0.000349524 i$ & $F_{\omega k_{y}}=-0.000118783 i$ \\
case & $F_{\omega k_{z}}=-0.000847166 i$ & $F_{k_{y} k_{y}}=-0.000065676 i$ \\
& $F_{k_{z} k_{z}}=0.000215422 i$ & $F_{k_{y} k_{z}}=0.000177085 i$ \\
& $\alpha_{1}=3.81986 \times 10^{-9} i \mu_{2}-3.20856 \times 10^{-6} i \delta \cos \left(\Omega_{0} T_{1}\right)-8.72807 \times 10^{-8} \Omega_{0} \delta \sin \left(\Omega_{0} T_{1}\right)$ \\
& $\alpha_{2}=-2.20145 \times 10^{-7}-4.50615 \times 10^{-7} i$ \\
\hline & $F_{\omega \omega}=-14.00444 \times 10^{-8} i$ & $F_{\omega k_{y}}=4.99548 \times 10^{-8} i$ \\
case & $F_{\omega k_{z}}=-2.68865 \times 10^{-7} i$ & $F_{k_{y} k_{y}}=-15.61828 \times 10^{-9} i$ \\
2 & $F_{k_{z} k_{z}}=16.38474 \times 10^{-8} i$ & $F_{k_{y} k_{z}}=3.73816 \times 10^{-8} i$ \\
& $\alpha_{1}=3.0639 \times 10^{-12} i \mu_{2}-9.0886 \times 10^{-10} i \delta \cos \left(\Omega_{0} T_{1}\right)-7.18915 \times 10^{-12} \Omega_{0} \delta \sin \left(\Omega_{0} T_{1}\right)$ \\
& $\alpha_{2}=-9.66417 \times 10^{-11}+3.91906 \times 10^{-9} i$ \\
\hline \multirow{4}{c}{ case } & $F_{\omega \omega}=6.6102 \times 10^{-8} i$ & $F_{\omega k_{y}}=-1.20357 \times 10^{-8} i$ \\
3 & $F_{\omega k_{z}}=1.23113 \times 10^{-7} i$ & $F_{k_{y} k_{y}}=2.30728 \times 10^{-9} i$ \\
& $F_{k_{z} k_{z}}=6.8073 \times 10^{-8} i$ & $F_{k_{y} k_{z}}=-1.19783 \times 10^{-8} i$ \\
& $\alpha_{1}=3.18955 \times 10^{-14} i \mu_{2}+4.24512 \times 10^{-11} i \delta \cos \left(\Omega_{0} T_{1}\right)+1.42821 \times 10^{-13} \Omega_{0} \delta \sin \left(\Omega_{0} T_{1}\right)$ \\
\hline \multirow{4}{*}{ case } & $\alpha_{2}=6.5857 \times 10^{-13}+1.33172 \times 10^{-11} i$ \\
4 & $F_{\omega \omega}=-0.00593664 i$ & $F_{\omega k_{y}}=0.00223118 i$ \\
& $F_{\omega k_{z}}=-0.0189051 i$ & $F_{k_{y} k_{y}}=-0.001679708 i$ \\
& $F_{k_{z} k_{z}}=-0.0081296 i$ & $F_{k_{y} k_{z}}=0.00439573 i$ \\
& $\alpha_{1}=1.08446 \times 10^{-7} i \mu_{2}-0.000122583 i \delta \cos \left(\Omega_{0} T_{1}\right)-8.36815 \times 10^{-6} \Omega_{0} \delta \sin \left(\Omega_{0} T_{1}\right)$ \\
& $\alpha_{2}=-0.0000442779-0.0000244122 i$ \\
\hline
\end{tabular}

instead of (41). These may be recast as the autonomous first-order system

$$
\begin{gathered}
\frac{d R}{d T_{1}}=R S \\
\frac{d \theta}{d T_{1}}=T \\
\frac{d S}{d T_{1}}=T^{2}+G_{0}+\bar{N}_{r} R^{2}-S^{2} \\
\frac{d T}{d T_{1}}=-2 S T+\bar{N}_{i} R^{2} .
\end{gathered}
$$

It is straightforward to verify that this system has no fixed points, so that, asymptotically in $T_{1}$, the solutions may (a) blow up in finite time (a so-called initial condition-dependent movable spontaneous singularity), when the perturbation expansions break down; (b) go to a periodic attractor or attracting limit cycle if any exists; (c) settle on a quasiperiodic attractor if there is one; or (d) exhibit bounded aperiodic dynamics with sensitivity to initial conditions and a positive Lyapunov exponent on a strange or a non-strange attractor, if it is a dissipative system for the given parameters. Hence, depending on the values of the parameters $G_{0}, \bar{N}_{r}$, and $\bar{N}_{i}$ and sometimes also the initial conditions, one may have varied, and complex, nonlinear evolution of the solution $A$ (of (37) or (40)). 
In this context, it is worth noting that one may decompose the spatially uniform version of (40) into real and imaginary parts in an alternative manner by writing $A \equiv A_{r}+i A_{i}$ (instead of the polar form $A \equiv R e^{i \theta}$ ), yielding the system

$$
\begin{gathered}
\frac{d A_{r}}{d T_{1}}=B_{1} \\
\frac{d A_{i}}{d T_{1}}=B_{2} \\
\frac{d B_{1}}{d T_{1}}=G_{0} A_{r}+\left(A_{r}^{2}+A_{i}^{2}\right)\left(A_{r} \bar{N}_{r}+A_{i} \bar{N}_{i}\right) \\
\frac{d B_{2}}{d T_{1}}=G_{0} A_{i}+\left(A_{r}^{2}+A_{i}^{2}\right)\left(A_{r} \bar{N}_{i}+A_{i} \bar{N}_{r}\right)
\end{gathered}
$$

which has fixed points

$$
\begin{aligned}
&\left(A_{r 0}, A_{i 0}\right)=(0,0) \\
&\left(A_{r 1,2}, A_{i 1,2}\right)=\left(\frac{ \pm \sqrt{G_{0}}}{\sqrt{2\left(\bar{N}_{i}-\bar{N}_{r}\right)}}, \frac{\mp \sqrt{G_{0}}}{\sqrt{2\left(\bar{N}_{i}-\bar{N}_{r}\right)}}\right) \\
&\left(A_{r 4,5}, A_{i 4,5}\right)=\left(\frac{ \pm i \sqrt{G_{0}}}{\sqrt{2\left(\bar{N}_{i}+\bar{N}_{r}\right)}}, \frac{ \pm i \sqrt{G_{0}}}{\sqrt{2\left(\bar{N}_{i}+\bar{N}_{r}\right)}}\right) \\
& B_{1}=0, \quad B_{2}=0 .
\end{aligned}
$$

One may analyze the real/imaginary nature and linear stability of these fixed points for various values of $G_{0}, \bar{N}_{r}$, and $\bar{N}_{i}$ in the standard way, as in Wollkind et al. (1994) or Roy Choudhury (1997) for instance. However, the analysis gets involved quite rapidly and in $\left(G_{0}, \bar{N}_{r}, \bar{N}_{i}\right)$ regimes where all fixed points are unstable, one again has the four possibilities for asymptotic dynamics listed above. We employ the equivalent polar form (44) in order to avoid this involved, though straightforward, analysis.

In order to illustrate the possible dynamics, which depends on the $\left(G_{0}, \bar{N}_{r}, \bar{N}_{i}\right)$ values and, possibly, on the initial conditions, we shall numerically integrate (44) with initial conditions

$$
\begin{array}{cl}
R(0)=0.1, & \theta(0)=0 \\
S(0)=-0.05, & T(0)=0.1 .
\end{array}
$$

Note that these are illustrative, and other initial conditions may be treated similarly. In each of our four numerical cases, the values of $\bar{N}_{r}$ and $\bar{N}_{i}$ are given in (42), while an appropriate $\mu_{2}$ is picked to give the chosen $G_{0}$.

Fig. 4 shows the behavior of the solution amplitude $R$ versus time for case 1 with $G_{0}=0.001$. The solutions blow up at $T_{1}=78.6814$ (an initial condition-dependent movable spontaneous singularity). By contrast, Fig. 5 , for case 1 and $G_{0}=-0.01$ (this figure and subsequent figures are for the $(R, S, T)$ plane), shows quasiperiodicity. (Note that the quasiperiodicity may be verified by the existence of irregularly spaced peaks in the numerical power spectral density (Hilborn, 1994; Nayfeh and Balachandran, 1995).)

By contrast, Figs. 6 and 7 , for case 2 with $G_{0}=0.01$ and $G_{0}=-0.01$ respectively, show chaotic solutions and quasiperiodicity respectively. The chaotic nature (bounded 


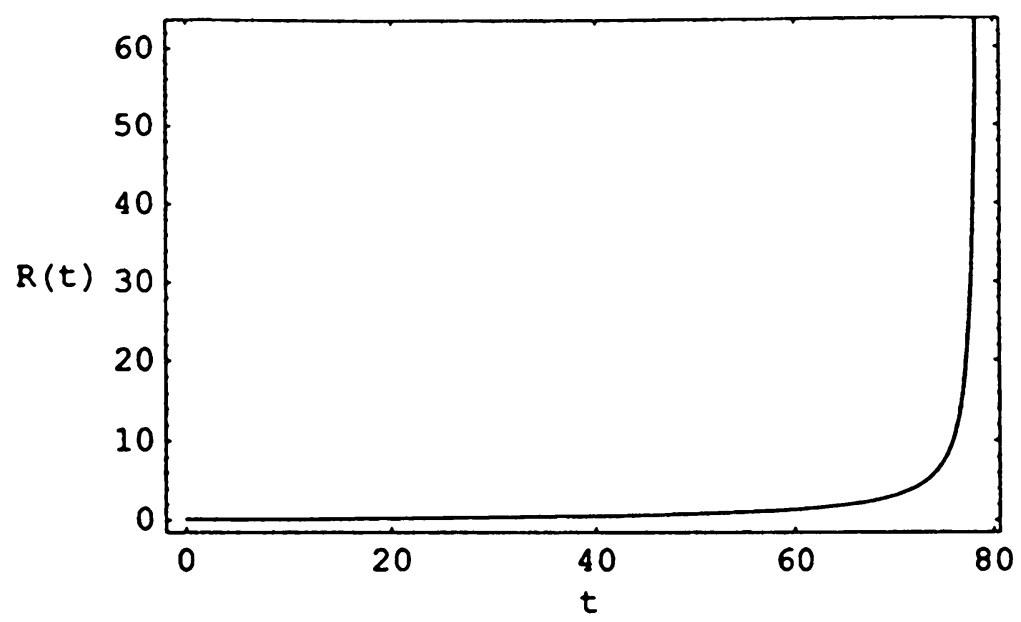

FIG. 4. The time evolution of the amplitude $R$ for case 1 ( $M F=$ $6, q_{D}=2.7, k_{y}=0.5, k_{z}=0.05$ (from Fig. 2)) with $G_{0}=0.001$

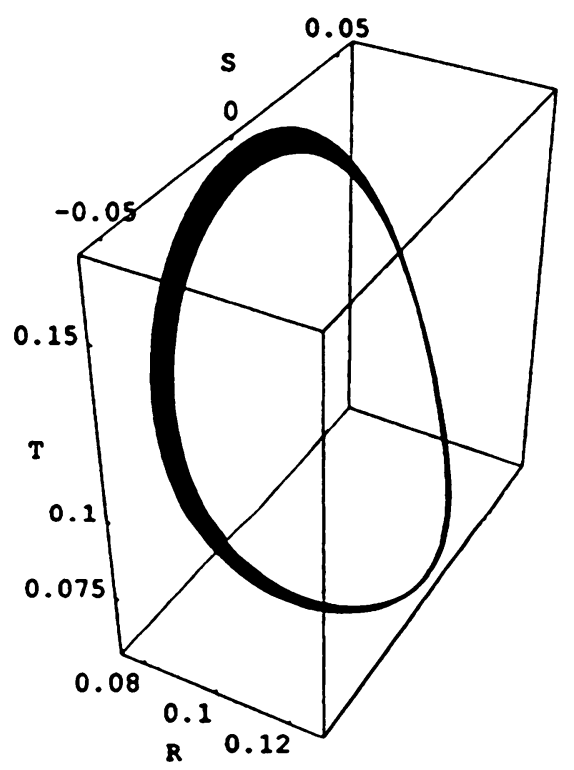

FIG. 5. The dynamics in the $(R, \theta, S)$ plane for case 1 with $G_{0}=-0.01$

aperiodic evolution with sensitivity to initial conditions and positive largest Lyapunov exponent) of the solution in Fig. 6 may be verified by the use of numerical diagnostics (Roy Choudhury, 1992; Hilborn, 1994; Nayfeh and Balachandran, 1995). In order to confirm this and further characterize the suspected chaotic solutions, we employ the standard numerical diagnostics, i.e., the power spectral density, the autocorrelation function, and the fractal dimensions (notice that one could also calculate Lyapunov exponents which 


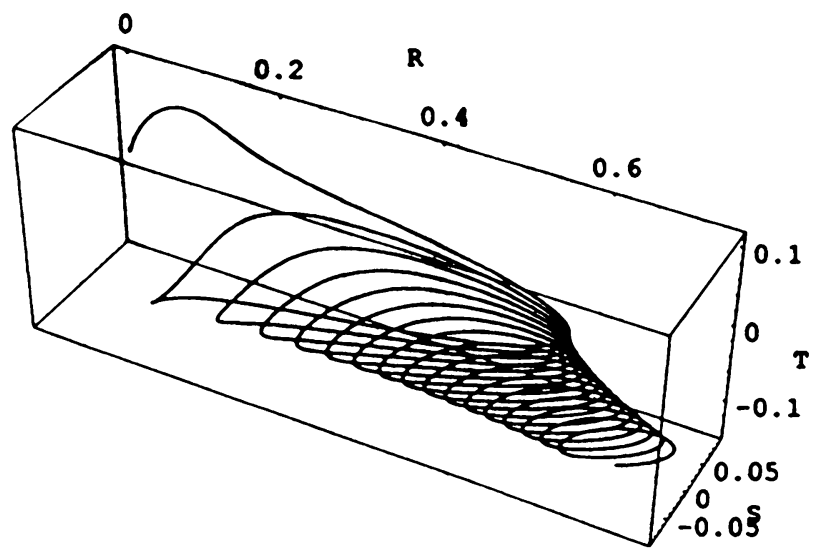

FIG. 6. The dynamics in the $(R, S, T)$ plane for case $2(M F=$ $2.4, q_{D}=0.5, k_{y}=0.5, k_{z}=0.05$ (from Fig. 2)) with $G_{0}=0.01$

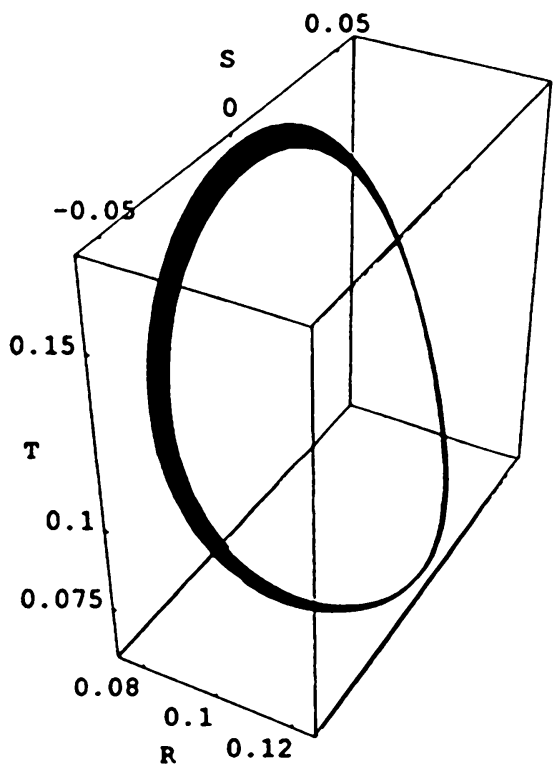

FIG. 7. As in Fig. 6 with $G_{0}=-0.01$

measure the rate of divergence of neighboring trajectories on an attractor). The autocorrelation function and the logarithm of the power spectral density of $R(t)$ are shown in Figs. 8 and 9. The "broad" features in the power spectral density plot in Fig. 9 are indicative of chaos or randomness, but one would strictly need to compare them with the width of peaks in the power spectrum of deterministic signals such as $\sin (t)$. However, the envelope of the correlation function decaying to zero in Fig. 8 is a definite indication of the solutions $R(t)$ being random. Sensitivity of the solutions to small changes in the initial conditions has also been verified. 


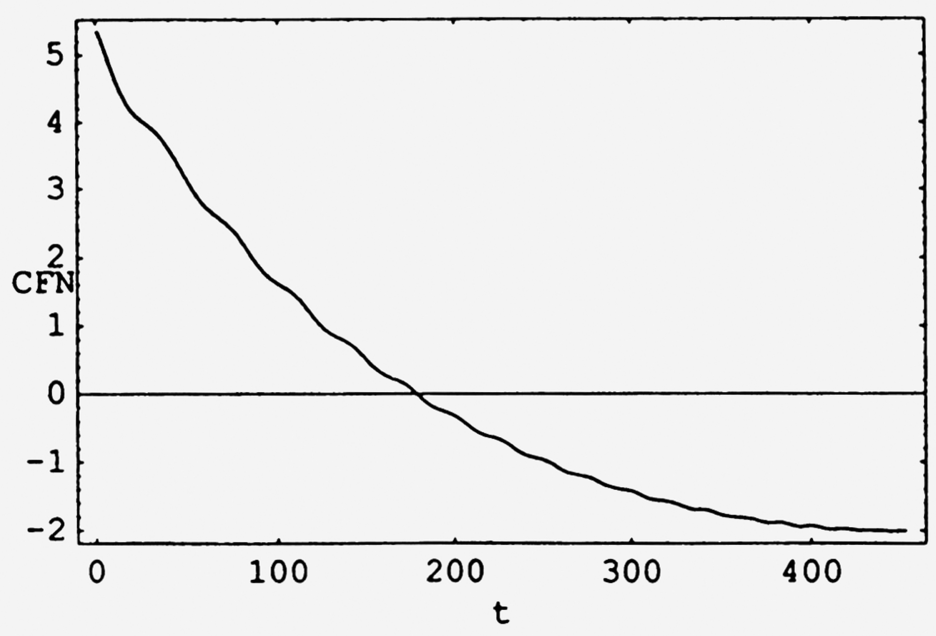

FIG. 8. The autocorrelation function as a function of time of the numerical time-series $R\left(T_{1}\right)$ of Fig. 6

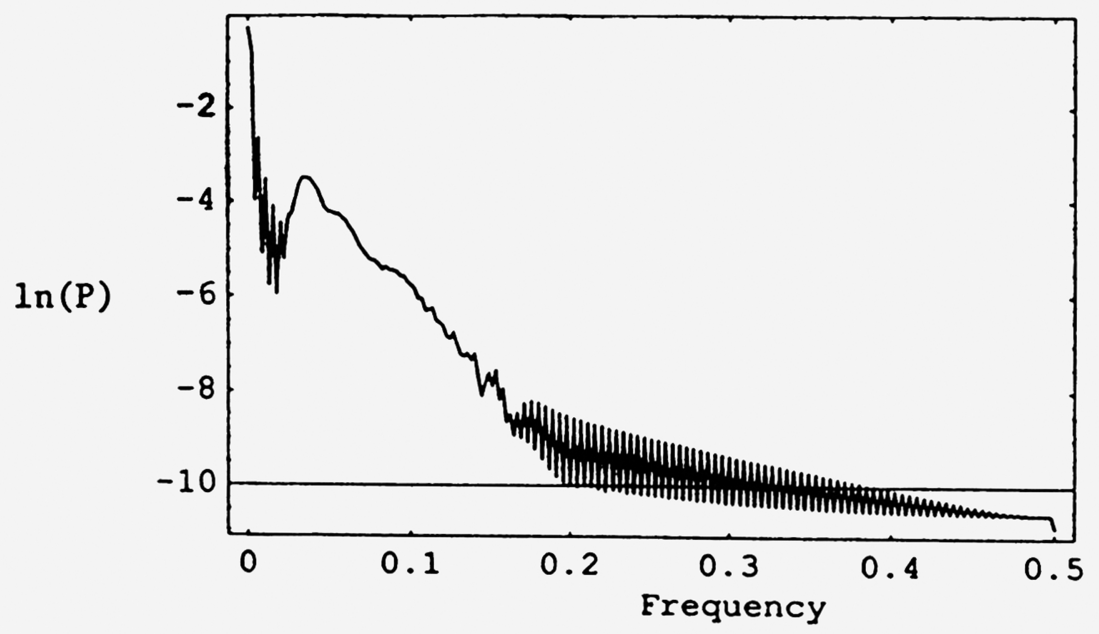

FIG. 9. The logarithm of the power spectral density $P$ of the numerical time-series $R\left(T_{1}\right)$ of Fig. 6 as a function of frequency $\nu$. Note the apparently "broad" features which are indicative of randomness. The approximate straight-line behavior at larger frequencies indicates a scaling law of the form $P=k_{1} \exp \left(-k_{2} \nu\right)$ which is characteristic of "weak" randomness with a small number of degrees of freedom, as opposed to "strong" randomness with a large number of degrees of freedom and $P=k_{1} \nu^{-k_{2}}$

However, we move on to a more quantitative and definitive numerical diagnostic, i.e., the fractal dimension, noting that the first zero of the correlation function is at $t_{c} \sim 180$. In order to distinguish low-dimensional (deterministic) chaos from strong randomness or stochastic behavior with many degrees of freedom, one computes the dimensions as 
discussed below. The correlation time $t_{c}$ will be employed in setting "delays" for the dimension calculations (Nayfeh and Balachandran, 1995; Hilborn, 1994). Of several possible alternative definitions for the fractal dimension, we employ the cluster fractal dimension $D$ of Termonia and Alexandrowicz, which is defined by

$$
n=k[R(n)]^{D}, \quad n \rightarrow \infty,
$$

where $R(n)$ is the average radius of an $E$-dimensional ball containing $n$ points. Thus, $D$ is the slope of a plot of $\log [n]$ versus $\log [R(n)]$. More usefully, if a scaling law (48) exists it would show up as a horizontal line on a plot of $d \log [n] / d \log [R(n)]$ versus $\log [n]$, with the height of the line being a measure of $D$. Numerical computation of $D$ is somewhat subtle, and requires the embedding of the numerical time-series $N(t)$ in $E$-dimensions with various values of $E$, via the construction (from $N(t))$ of " $E$-tuples":

$$
\begin{gathered}
\{[N(t), N(t+r), N(t+2 r), \ldots, N(t+r(E-1))], \\
[N(t+r), N(t+2 r), \ldots, N(t+E r)], \ldots\} .
\end{gathered}
$$

This is a consequence of the Takens embedding theorem (Nayfeh and Balachandran, 1995). For each choice of embedding dimension $E$, the "delay" $\tau$ is typically chosen to be $t_{c}, \frac{t_{c}}{2}$, and $2 t_{c}$ and $D$ is estimated for each $\tau$ from the slope of a $\log [n] / \log [R(n)]$ plot (provided this is approximately a straight line, or there is a well-defined scaling region) or the height of an approximate horizontal straight line in a $\frac{d \log [n]}{d \log [R(n)]} / \log [n]$ plot. This is repeated for increasing $E$ until the estimates for $D$ saturate, which yields the converged estimate for $D$ (provided well-defined straight lines or scaling regions (48) occur in the plots and the $D$ values obtained for $\tau=t_{c}, \frac{t_{c}}{2}$, and $2 t_{c}$ are in good agreement). Following this procedure, a typical sample plot is shown in Fig. 10.

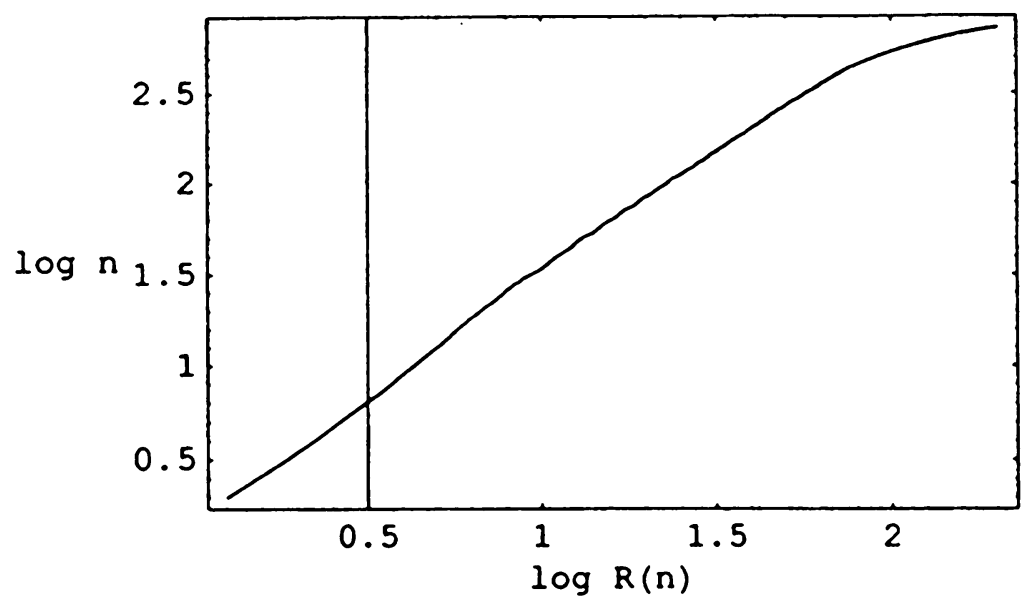

FIG. 10. Plot of $\log [n]$ versus $\log [R(n)]$ from the cluster dimension calculation for the time-series of Fig. 6. The embedding dimension is $E=2$, and the delay is $\tau=t_{c}=180$

Fig. 10 shows the $\log [n] / \log [R(n)]$ plot for $E=2$ and $\tau=t_{c}=180$, which is an approximate straight line. From the slope of the approximate straight line in Fig. 10 (the 
well-defined straight line indicates well-defined scaling regions), we may estimate the converged cluster fractal dimensions to be approximately in the range $D \in(1.53,1.59)$, confirming bounded low-dimensional (deterministic) chaotic solutions evolving on a strange attractor with fractal dimension $D \in(1.53,1.59)$.

Fig. 11, for case 3 with $G_{0}=-0.01$, again shows quasiperiodic dynamics. However, Figs. 12 and 13 , for case 4 with $G_{0}=-0.005$ and $G_{0}=0.005$, show spontaneous initial condition-dependent singularities at $T_{1}=777.74$ and $T_{1}=47.1465$ respectively. Of these, Fig. 12 shows an interesting evolution of the orbits prior to the blow up. Notice that the system is not dissipative for the parameters used, hence the eventual blow up of the solution.

(b) Nonlinear traveling wave envelopes

Next, we consider spatial dependence of solutions. Neglecting the $y$ variation, Eq. (40) becomes

$$
\frac{\partial^{2} A}{\partial T_{1}^{2}}-\omega_{k_{z}}^{2} \frac{\partial^{2} A}{\partial Z^{\prime 2}}=G A+\bar{N}|A|^{2} A .
$$

Note that $\omega_{k_{z}}^{2}$ may be computed in each of our numerical cases by using (39) and Table 1. Considering traveling wave solutions of the form $A=A(\bar{Z}) \equiv A\left(Z^{\prime}-V T_{1}\right)$ gives

$$
\left(V^{2}-\omega_{k_{z}}^{2}\right) \frac{\partial^{2} A}{\partial \bar{Z}^{2}}=G A+\bar{N}|A|^{2} A .
$$

For $\bar{N}$ real, one may take $A$ to be real and find solutions for different signs of $G$ and $\bar{N}$ in terms of elliptic functions (Weissman, 1979; Pedlosky, 1972). However, for our complex $\bar{N}$, we shall employ a different approach, similar to that employed above in Sec. 6a.

Writing $A=R e^{i \theta}$ as before, it is straightforward to recast (50) into the first-order system

$$
\begin{gathered}
R^{\prime}=R S \\
\theta^{\prime}=T \\
S^{\prime}=T^{2}+G_{0 *}+\bar{N}_{r *} R^{2}-S^{2} \\
T^{\prime}=-2 S T+\bar{N}_{i *} R^{2},
\end{gathered}
$$




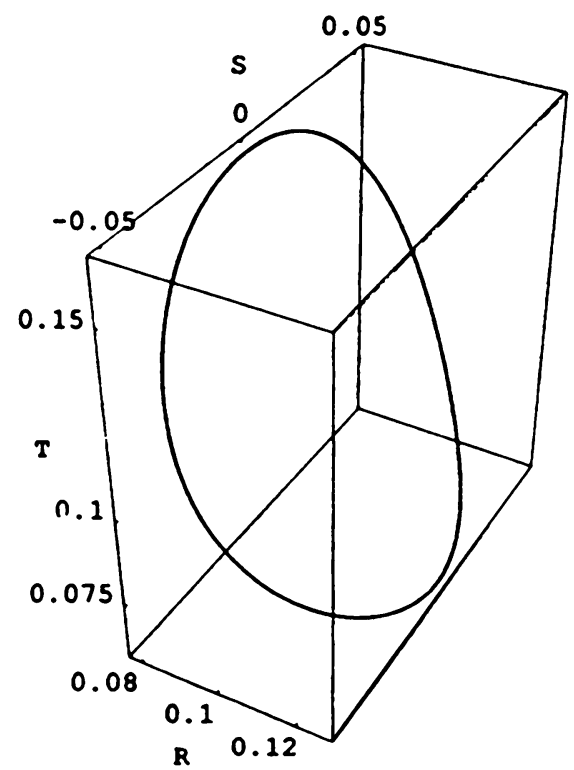

FIG. 11. The dynamics in the $(R, S, T)$ plane for case $3(M F=$ $2, q_{D}=0.9, k_{y}=0.5, k_{z}=0.05$ (from Fig. 3)) with $G_{0}=-0.01$

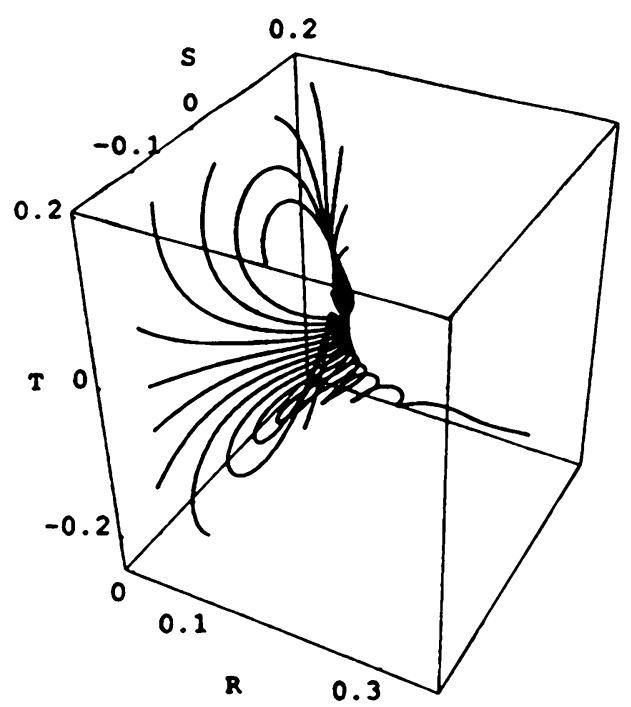

FIG. 12. The dynamics in the $(R, S, T)$ plane for case $4(M F=$ $9, q_{D}=4, k_{y}=0.5, k_{z}=0.05$ (from Fig. 3)) with $G_{0}=-0.005$ 


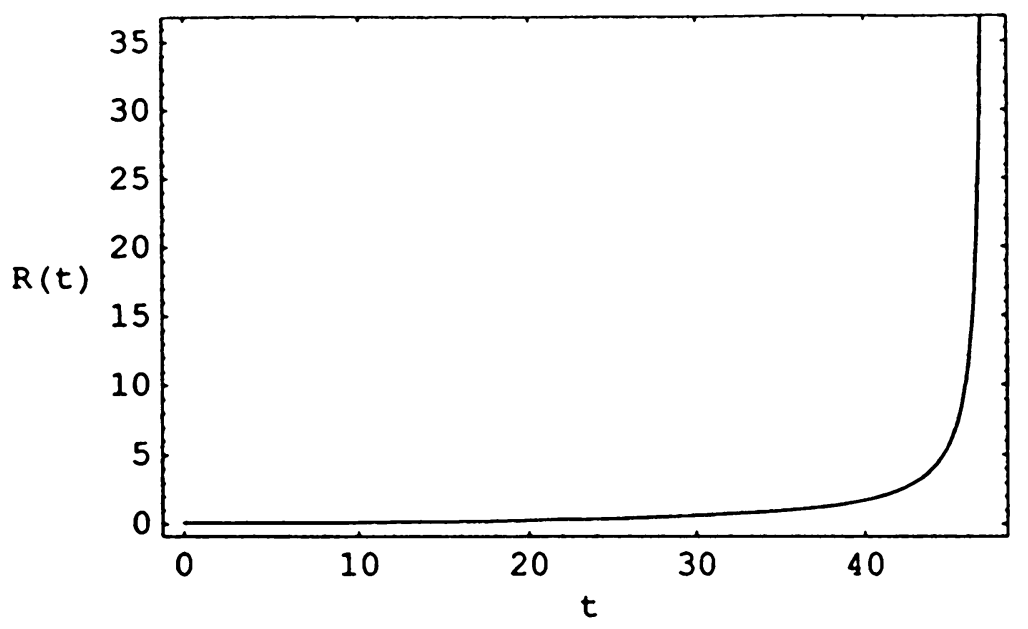

FIG. 13. The time evolution of the amplitude $R$ for case 4 with $G_{0}=0.005$

where the prime denotes a derivative with respect to $\bar{Z}$ and the new parameters are

$$
\begin{aligned}
G_{0 *} & =\frac{G_{0}}{\left(V^{2}-\omega_{k_{z}}^{2}\right)}, \\
\bar{N}_{r *} & =\frac{\bar{N}_{r}}{\left(V^{2}-\omega_{k_{z}}^{2}\right)}, \\
\bar{N}_{i *} & =\frac{\bar{N}_{i}}{\left(V^{2}-\omega_{k_{z}}^{2}\right)} .
\end{aligned}
$$

The system (51) is identical to system (44) treated earlier, save for the new parameters given by (52). Thus it may be treated in similar fashion in any of our cases, obtaining $G_{0}, \bar{N}_{r}$, and $\bar{N}_{i}$ using (42), picking a traveling wave speed, and computing $\omega_{k_{z}}^{2}$ in each case using (39) and Table 1. As an illustrative example, we pick case 2 in (42b), when $\omega_{k_{z}}=1.56172$. Choosing the traveling wave speed $V=1$, the traveling wave solutions of (51) show quasiperiodic behavior as shown in Fig. 14.

We may consider other cases (and different traveling wave speeds $V$ in each case). Given the similarity of (51) to (44) and the discussion of the latter in Sec. 6a, it is clear that the traveling wave solutions of (50) may exhibit blow up in finite time, or periodic, quasiperiodic, or chaotic evolution.

In earlier cases (Weissman, 1979), nonlinear envelope solutions (of permanent form) of $(49)$ in the most interesting case $(G>0, \bar{N}$ (real) $<0)$ had amplitudes evolving to permanent waveforms given in terms of the $s n$ and $d n$ elliptic functions. These solutions were somewhat analogous to the final permanent waveforms considered by Newell (1974) for the Ginzburg-Landau (Newell-Whitehead) canonical equation in the alternate setting of dissipative instabilities. In our case, we may have periodic, quasiperiodic, or chaotic waveforms for the supersonic Kelvin-Helmholtz instability with general anisotropy. Such permanent waveforms may correspond to trains of vortices for the present supersonic 


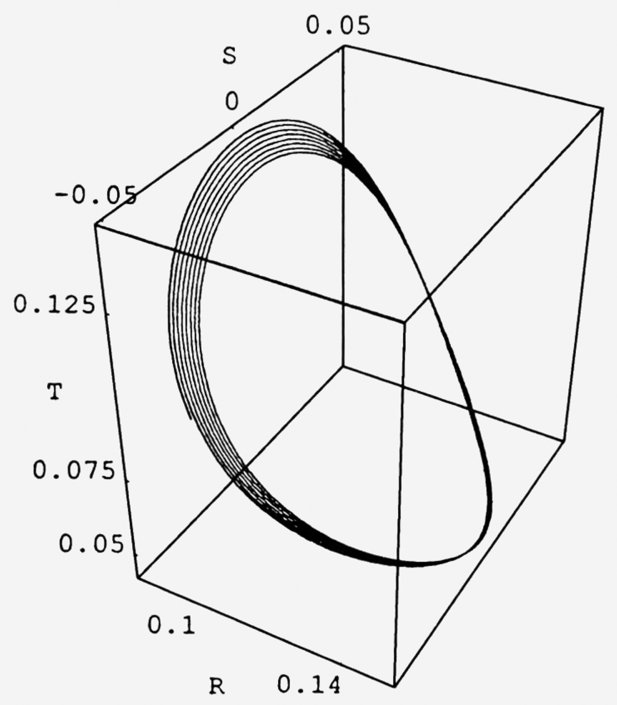

FIG. 14. The dynamics in the $(R, S, T)$ plane for case $3(M F=$ $2, q_{D}=0.9, k_{y}=0.5, k_{z}=0.05$ (from Fig. 3)) where $\omega_{k_{z}}=1.56172$ and the traveling wave speed $V=1$

instability. The different evolutions for $x>0, x<0$ seen in (35) would imply two families of such vortices on the two sides of the original discontinuity at $x=0$. Similar behavior would exist for most regimes of case (a), where both $G$ and $\bar{N}$ (the linear and nonlinear terms) are stabilizing, save for the narrow unstable wedge in the $(k, B)$ plane found by Parkes. For subsonic two-dimensional shear layers, there is detailed experimental (Brown and Roshko, 1974; Winant and Browand, 1974), and simulation (Aref and Siggia, 1981) information on the nonlinear evolution, giving rise to an ensemble of plus and minus vortices (somewhat like the von Karman vortex sheet). There are less conclusive indications of similar ensembles of vortices in simulations for the supersonic case (Norman et al., 1982). Subsequent investigations will seek to correlate nonlinear analyses of the supersonic instability for more realistic spatially non-uniform equilibria relevant to space and astrophysical configurations to the results of detailed simulations.

(c) Forced System

Next, we consider the possibility of the occurrence of chaotic solutions due to the external forcing or modulation of the magnetic field at frequency $\Omega_{0}$. We shall assume that the static magnetic field is supercritical; i.e., linear instability has occurred and shall look for the conditions on the modulation amplitude $\delta$ for which chaotic solutions may occur. We shall use the numerical diagnostics employed in Secs. 6a and 6b.

Neglecting spatial modulations, we have

$$
\frac{\partial^{2} A}{\partial T_{1}^{2}}-G_{0} A-\bar{N} A^{3}+\xi \frac{\partial A}{\partial T_{1}}=\left[\beta_{1} \cos \left(\Omega_{0} T_{1}\right)+\beta_{2} \sin \left(\Omega_{0} T_{1}\right)\right] A .
$$

Here,

$$
G \equiv G_{0}+\beta_{1} \cos \left(\Omega_{0} T_{1}\right)+\beta_{2} \sin \left(\Omega_{0} T_{1}\right)
$$


and we have added a damping term to simulate the effect of any form of dissipation such as viscosity (Newton, 1988; Malik and Singh, 1993). Note that $G_{0}, \beta_{1}$, and $\beta_{2}$ are given for the four cases by (45). Using stretched variables

$$
\begin{aligned}
X & \equiv\left(-\frac{\bar{N}}{G_{0}}\right)^{\frac{1}{2}} A, \\
\tau & \equiv \sqrt{G_{0}} T_{1},
\end{aligned}
$$

(55) may be rewritten as the first-order dynamical system (for $x_{1} \equiv X, x_{2} \equiv \frac{d X}{d \tau}$ ):

$$
\begin{gathered}
\frac{d x_{1}}{d \tau}=x_{2} \\
\frac{d x_{2}}{d \tau}=x_{1}-x_{1}^{3}+\chi\left[\beta_{1} x_{1} \cos \phi+\beta_{2} x_{1} \sin \phi-\beta_{3} x_{2}\right] \\
\frac{d \phi}{d \tau}=\Omega .
\end{gathered}
$$

Here,

$$
\Omega \equiv \frac{\Omega_{0}}{\sqrt{G_{0}}}, \quad \beta_{3} \equiv \xi \sqrt{G_{0}}
$$

and

$$
\chi \equiv \frac{1}{G_{0}},
$$

and $\chi$ is a measure of the strength of the forcing and damping terms.

For all of our cases in (42), $\beta_{1}$ and $G_{0}$ are real, while $\beta_{2} \equiv i \beta_{2 i}$ is pure imaginary. Hence, $\tau$ and $\beta_{3}$ are real from (56) or (58). Equations (57) may now be recast into the following system of equations where $x_{1}=x_{1 r}+i x_{1 i}$, and $x_{2}=x_{2 r}+i x_{2 i}$ :

$$
\begin{gathered}
\frac{d x_{1 r}}{d \tau}=x_{2 r}, \\
\frac{d x_{1 i}}{d \tau}=x_{2 i}, \\
\frac{d x_{2 r}}{d \tau}=x_{1 r}-x_{1 r}^{3}+3 x_{1 i}^{2} x_{1 r}+\chi\left[\beta_{1} x_{1 r} \cos \phi-\beta_{2 i} x_{1 i} \sin \phi-\beta_{3} x_{2 r}\right], \\
\frac{d x_{2 i}}{d \tau}=x_{1 i}+x_{1 i}^{3}-3 x_{1 i} x_{1 r}^{2}+\chi\left[\beta_{1} x_{1 i} \cos \phi+\beta_{2 i} x_{1 r} \sin \phi-\beta_{3} x_{2 i}\right], \\
\frac{d \phi}{d \tau}=\Omega .
\end{gathered}
$$

To illustrate some representative dynamical behavior of this forced system, we integrate (60) with initial conditions

$$
\begin{gathered}
x_{1 r}(0)=x_{1 i}(0)=0.1 \\
x_{2 r}(0)=0, \quad x_{2 i}(0)=0.2
\end{gathered}
$$

and parameters corresponding to Fig. 6, i.e., case 2 with $G_{0}=0.01$. The resulting plot of $x_{1 r}$ is shown in Fig. 15. Figures 16 and 17 show the power spectral density and autocorrelation function of the $x_{1 r}$ time-series in Fig. 15. The very broad features in the power spectral density and the envelope of the autocorrelation going to zero again indicate that the solutions are chaotic. The solutions of the forced system (60) may clearly be 
investigated in an analogous manner for the various other cases in (42). However, we omit the details for the sake of brevity.

In conclusion, we have comprehensively analyzed the weakly nonlinear evolution of the supersonic Kelvin-Helmholtz instability of velocity shear layers with general anisotropy. We find spatially uniform solutions as well as traveling wave solutions exhibiting dynamics significantly more complex than in earlier cases of instabilities of the "dispersive" variety governed by a nonlinear Klein-Gordon equation. Subsequent work will seek to use these results in modeling various space and astrophysical configurations containing supersonic velocity shear layers.

Acknowledgment. The authors wish to express their sincere gratitude to an anonymous referee whose comments significantly improved the paper. In particular, we are grateful for the detection of an error in Sec. 6c and suggestions which improved the discussion of the solutions in all of Sec. 6 .

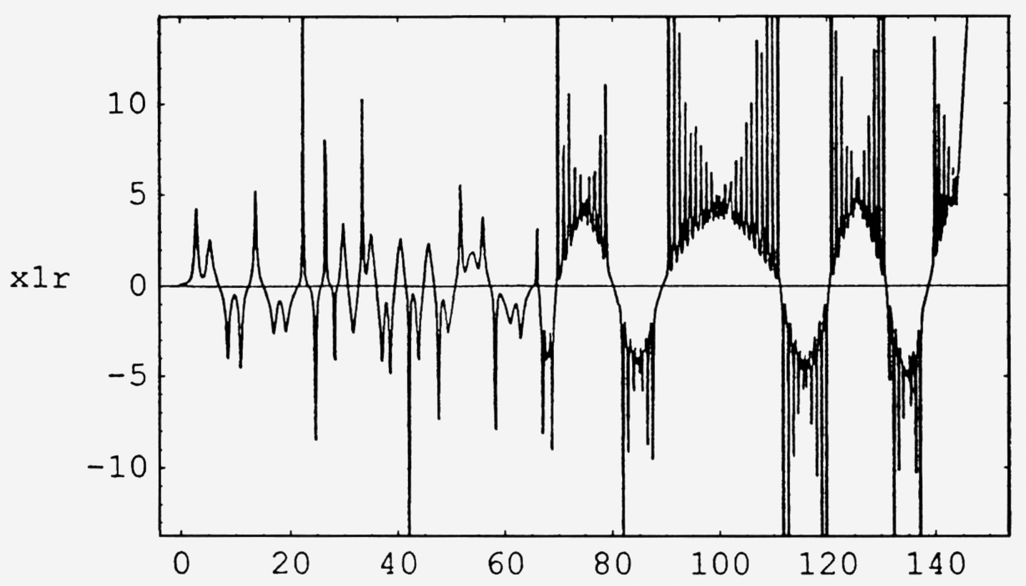

FIG. 15. The time evolution of $x_{1 r}$ in (60) for case 2 with $G_{0}=0.01$ 


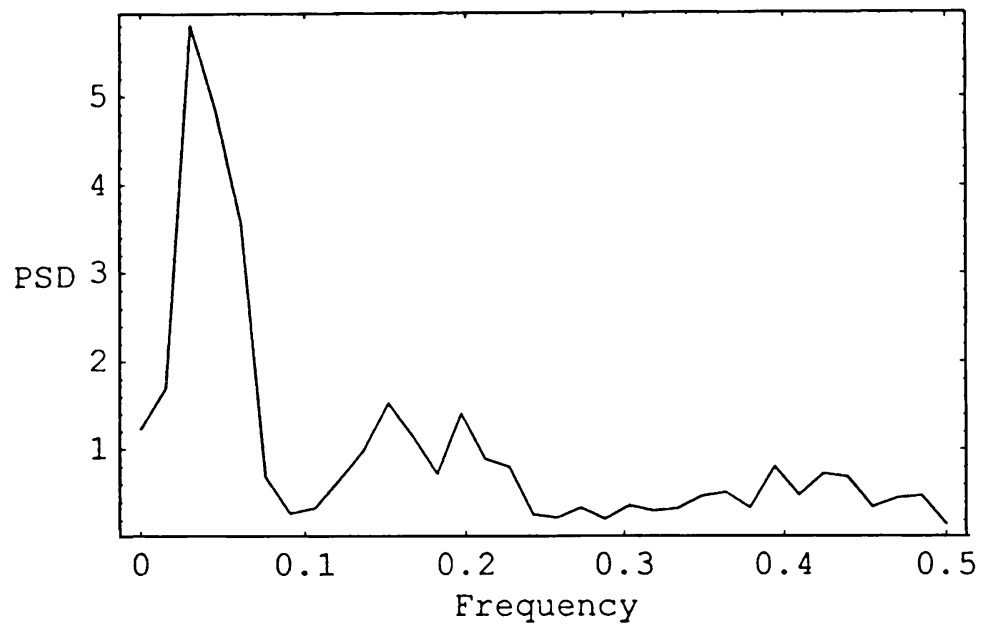

Fig. 16. The power spectral density of the time-series for $x_{1 r}$ in Fig. 15. Note the very broad features

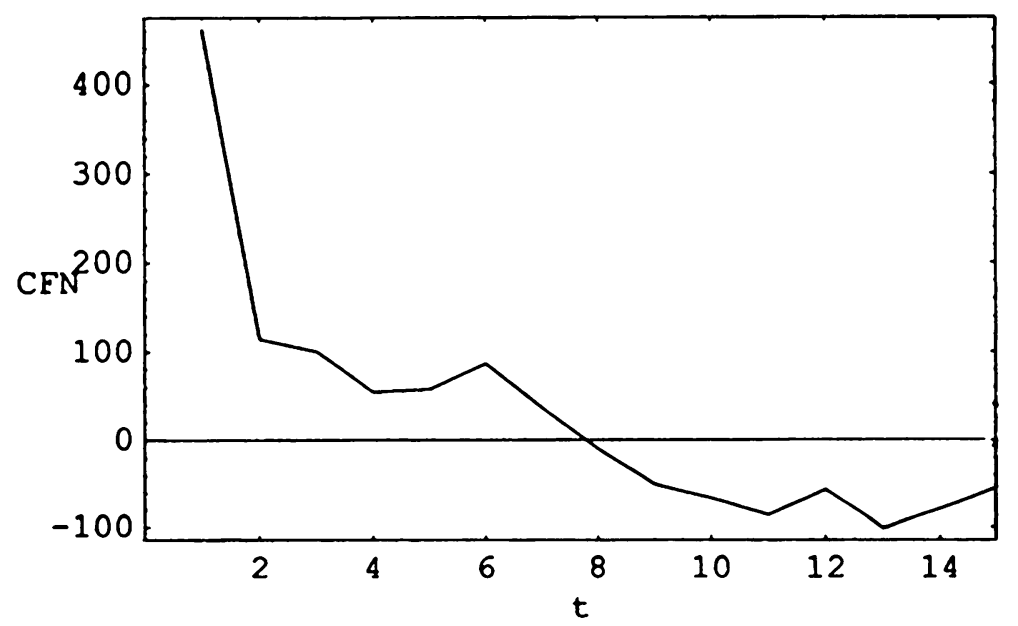

FIG. 17. The autocorrelation function versus time for the time-series in Fig. 15. Note that it goes to zero at $t \approx 7.75$

\section{REFERENCES}

[1] Ablowitz, M. J., and Segur, H. (1981). "Solitons and the Inverse Spectral Transform." Philadelphia: SIAM.

[2] Abraham-Shrauner, B. (1973). "Small Amplitude Hydromagnetic Waves for a Plasma with a Generalized Polytrope Law." Plasma Phys. 15, 375-385.

[3] Abramowitz, M., and Stegun, I. A. (1964). "Handbook of Mathematical Functions." Washington: National Bureau of Standards.

[4] Aref, H., and Siggia, E. D. (1981). "Evolution and Breakdown of a Vortex Street in Two Dimensions." Journal of Fluid Mechanics 109, 435-463. 
[5] Begelman, M. C., Blandford, R. D., and Rees, M. J. (1984). "Theory of Extragalactic Radio Sources". Review of Modern Physics 56, 255-351.

[6] Benney, D. J., and Newell, A. C. (1967). Journal of Math and Physics 46, 363.

[7] Benney, D. J., and Roskes, G. J. (1969). Studies in Applied Math. 48, 377.

[8] Blandford, R. D., and Pringle, J. E. (1976). "Kelvin-Helmholtz Instability of Relativistic Beams." Monthly Notices of the Royal Astronomical Society 176, 443-454.

[9] Blumen, W. (1970). "Shear Layer Instability of an Inviscid Compressible Fluid." Journal of Fluid Mechanics 40, 769-781.

[10] Blumen, W., Drazin, P. G., and Billings, D. F. (1975). "Shear Layer Instability of an Inviscid Compressible Fluid, Part 2." Journal of Fluid Mechanics 71, 305-316.

[11] Brandt, J. C., and Mendis, D. A. (1979). The Solar Wind, in "Solar System Plasma Physics." (C. F. Kennel, L. Lanzerotti, and E. N. Parker, Eds.). Amsterdam: North Holland.

[12] Bridge, H. S., Belcher, J. W., Lazarus, A. J., Sullivan, J. D., Bagenal, F., McNutt, Jr., R. L., Oglivie, K. W., Scudder, J. D., and Sittler, E. C. (1979). "Plasma observations near Jupiter: Initial results". Science 206, 972-976.

[13] Brown, G. L., and Roshko, A. (1974). "On density effects and large structure in turbulent mixing layers." Journal of Fluid Mechanics 64, 775-816.

[14] Brown, K. G., and Roy Choudhury, S. (2002). "An analytical study of the Kelvin-Helmholtz instabilities of compressible magnetized tangential velocity discontinuities with generalized polytrope laws." Quart. Appl. Math. 60, 601-630.

[15] Brown, K. G., and Roy Choudhury, S. (2002). "The initial value problem for the Kelvin-Helmholtz instabilities of high velocity magnetized shear layers with generalized polytrope laws", Quart. Appl. Math. 60, 657-673.

[16] Chandrasekhar, S. (1981). "Hydrodynamic and Hydromagnetic Stability." New York: Dover (originally published 1961, Oxford Univ. Press).

[17] Craik, A. D. D., Nagata, M., and Moroz, I. M. (1992). "Second Harmonic Resonance in Nonconservative Systems." Wave Motion 15, 173.

[18] Craik, A. D. D. (1985). "Wave Interactions and Fluid Flows." Cambridge: Cambridge Univ. Press.

[19] Davey, A., and Stewartson, K. (1974). "On three-dimensional packets of surface waves." Proc. of the Royal Society A338, 101.

[20] Dobrowolny, H. and D'Angelo, N. (1972). "Wave motion in type I comet tails." Cosmic Plasma Physics (K. Schindler, ed.), New York: Plenum.

[21] Dodd, R. K., Eilbeck, J. C., Gibbon, J. D., and Morris, H. C. (1982). "Solitons and Nonlinear Wave Equations." London: Academic Press.

[22] Drazin, P. G. (1970). Journal of Fluid Mechanics 42, 321.

[23] Drazin, P. G. and Reid, W. H. (1981). "Hydrodynamic Stability." Cambridge: Cambridge Univ. Press.

[24] Ershkovich, A. I., Nusnov, A. A., and Chernikov, A. A. (1972). "Oscillations of type I comet tails." Planetary and Space Science 20, 1235-1243.

[25] Ershkovich, A. I., and Chernikov, A. A. (1973). "Nonlinear waves in type I comet tails." Planetary and Space Science 21, 663-673.

[26] Fejer, J. A. (1964). "Hydromagnetic stability at a fluid velocity discontinuity between compressible fluids." Physics of Fluids 7, 499-503.

[27] Gerwin, R. A. (1968). "Stability of the Interface between two fluids in relative motion." Rev. Modern Phys. 40, 652-658.

[28] Gibbon, J. D., James, I. N., and Moroz, I. (1979). "The Sine-Gordon equation as a model for a rapidly rotating baroclinic fluid." Proc. Roy. Soc. London Ser. A 367, 219.

[29] Gibbon, J. D., and McGuinness, M. J. (1981). "Amplitude equations at the critical points of unstable dispersive physical systems." Proc. Roy. Soc. London Ser. A 377, 185.

[30] Hasimoto, H., and Ono, H. (1972). Journal of the Physical Society of Japan 33, 805.

[31] Hilborn, R. C. (1994). "Chaos and Nonlinear Dynamics." New York: Oxford Univ. Press.

[32] Infeld, E., Ziemkiewicz, J., and Rowlands, G. (1987). "Stability of Nonlinear Hydromagnetic Waves and Solitons." Physics of Fluids 30, 2330.

[33] Infeld, E., and Rowlands, G. (1979). "Stability of nonlinear ion sound waves and solitons in plasmas." Proc. Roy. Soc. London Ser. A 366, 537.

[34] Jokipii, J. R., and Davis, L. (1969). "Long wavelength turbulence and the heating of the solar wind." Astrophys. Journal 156, 1101-1106. 
[35] Kaup, D. J., Roy Choudhury, S., and Thomas, G. E. (1988). "The full second-order theory of the diocotron and magnetron resonances." Phys Rev. A 38, 1402.

[36] Landau, L. D. (1944). "The instability of moving superposed fluids." Akad. Nauk SSSR C. R. Dokl. 44, 139-144.

[37] Lange, C. G., and Newell, A. C. (1971). "The post buckling problem for thin elastic shells." SIAM Journal of Applied Math. 21, 605.

[38] Lange, C. G., and Newell, A. C. (1974). "A stability criterion for envelope equations." SIAM Journal of Applied Math. 27, 441.

[39] Lerche, I. (1966). "Validity of the hydromagnetic approach in discussing instability of the Magnetospheric Boundary." Journal of Geophys. Res. 71, 2365-2371.

[40] Michalke, A. (1964). "On the inviscid instability of the hyperbolic-tangent velocity profile." Journal of Fluid Mechanics 19, 543-556.

[41] Miles, J. W. (1957). "On wind over water." Journal Acoust. Soc. Amer. 29, 226-230.

[42] Miles, J. W. (1958). "On the disturbed motion of a plane vortex sheet." Journal of Fluid Mechanics $4,538-552$.

[43] Miura, A., and Pritchett, P. L. (1982). "Nonlocal stability analysis of the MHD Kelvin-Helmholtz instability in a compressible plasma." Journal of Geophy. Res. 87, 7431-7444.

[44] Miura, A. (1984). "Anomalous transport by magnetohydrodynamic Kelvin-Helmholtz instabilities in the solar wind-magnetosphere interaction." Journal of Geophy. Res. 89, 801-818.

[45] Murakami, Y. (1986). "A note on the modulational instability of the Klein-Gordon equation." Journal Phys. Soc. Japan 55, 3851.

[46] Nayfeh, A. H., and Balachandran, B. (1995). "Applied Nonlinear Dynamics." New York: Wiley.

[47] Nayfeh, A. H., and Saric, W. S. (1971). "The Kelvin-Helmholtz Instability I." Journal of Fluid Mechanics 46, 209.

[48] Nayfeh, A. H., and Saric, W. S. (1972). "The Kelvin-Helmholtz Instability II." Journal of Fluid Mechanics 55, 311.

[49] Nayfeh, A. H. (1973). "Perturbation Methods." New York: Wiley.

[50] Nepveu, M. (1980). "Cylindrical jets." Astronom. and Astrophys. 84, 14-21.

[51] Ness, N. F., Acuna, M. H., Lepping, R. P., Connerney, J. E. P., Behannon, K. W., and Burlaga, L. F. (1981). "Magnetic field studies by Voyager I." Science 212, 211-217.

[52] Newell, A. C., and Whitehead, J. A. (1969). "Finite bandwidth, finite amplitude convection." Journal of Fluid Mechanics 38, 279.

[53] Newell, A. C. (1974). "Nonlinear wave motion." Lect. Appl. Math. 15, 157.

[54] Newton, P. K. (1988). "Chaos in Rayleigh-Benard Convection with external driving." Physics Review A37, 932.

[55] Norman, M. L., Smarr, L., Winkler, K. H. A., and Smith, M. D. (1982). "Instabilities of cylindrical jets." Astronom. and Astrophys. 113, 285-351.

[56] Ochoa, F. L., and Murray, J. D. (1983). "A nonlinear analysis for spatial structure in a reactiondiffusion model." Bull. Math. Biol. 45, 917.

[57] Parker, E. N. (1963). "Interplanetary Dynamical Processes." New York: Interscience.

[58] Parkes, E. J. (1991). "Modulational instability in the Klein-Gordon equation." Wave Motion 13, 261.

[59] Pearlstein, L. D., and Berk, H. L. (1969). "Bound states of a Schrödinger equation." Phys. Rev. Lett. 23, 220.

[60] Pedlosky, J. (1970). "Finite amplitude baroclinic waves." Journal Atmospheric Science 27, 15.

[61] Pedlosky, J. (1972). "Finite amplitude baroclinic wave packets." Journal Atmospheric Science 29, 680.

[62] Pritchett, P. L., and Coroniti, F. V. (1984). "The collisionless macroscopic Kelvin-Helmholtz instability I. Transverse electrostatic mode." Journal Geophys. Res. 89, 168-178.

[63] Pu, Z. Y., and Kivelson, M. G. (1983). "Kelvin-Helmholtz instability at the magnetopause: Solution for compressible plasmas." Journal Geophys. Res. 88, 841-852 and "Energy flux into the magnetosphere," 853-861.

[64] Ray, T. P. (1982). "The effects of a simple shear layer on the growth of Kelvin-Helmholtz instabilities." Monthly Notices Roy. Astronom. Soc. 198, 617-625.

[65] Ray. T. P., and Erschkovich, A. I. (1983). "Kelvin-Helmholtz instabilities of magnetized shear layers." Monthly Notices Roy. Astronom. Soc. 204, 821-826. 
[66] Roy Choudhury, S., and Lovelace, R. V. E. (1986). "On the Kelvin-Helmholtz instabilities of high-velocity magnetized shear layers." Astrophys. Journal 302, 188-199.

[67] Roy Choudhury, S. (1986). "Kelvin-Helmholtz instabilities of supersonic magnetized shear layers." Journal Plasma Phys. 35, 375-392.

[68] Roy Choudhury, S., and Patel, V. L. (1985). "Kelvin-Helmholtz instabilities of high-velocity, magnetized anisotropic shear layers." Physics of Fluids 28, 3292-3301.

[69] Roy Choudhury, S. (1990). "Global asymptotic analysis of the Kelvin-Helmholtz instability of supersonic shear layers." Canad. Journal Phys. 68, 334-342.

[70] Roy Choudhury, S. (1992). Chaos, Solitons and Fractals 2, 393.

[71] Roy Choudhury, S. (1997). in "Nonlinear Instability Analysis" (Debnath, L., and Roy Choudhury, S., Eds.). Southampton: Computational Mechanics Publishers.

[72] Sen, A. K. (1965). "Stability of the magnetosphere boundary." Planet. Space Sci. 13, 131-141.

[73] Sen, A. K. (1964). "Effect of compressibility on Kelvin-Helmholtz instability in a plasma." Physics of Fluids 7, 1293-1298.

[74] Southwood, D. J. (1968). "The hydromagnetic stability of the magnetospheric boundary." Planet Space Sci. 16, 587-605.

[75] Southwood, D. J. (1974). "Some features of the field line resonances in the magnetosphere." Planet Space Sci. 22, 483-491.

[76] Stewartson, K., and Stuart, J. T. (1971). "Nonlinear stability of plane Poiseuille flow." Journal Fluid Mech. 48, 529 .

[77] Stuart, J. T. (1960). "On the nonlinear mechanics of wave disturbances in stable and unstable parallel flows." Journal Fluid Mech. 8, 183.

[78] Sturrock, P. A., and Hartle, R. E. (1966). "Two-fluid mode of the solar wind." Phys. Rev. Lett. $16,628-631$.

[79] Tajima, T., and Leboeuf, J. N. (1980). "Kelvin-Helmholtz instability in supersonic and superAlfvénic fluids." Physics of Fluids 23, 884-888.

[80] Talwar, S. P. (1965). "Kelvin-Helmholtz instability in an anisotropic plasma." Physics of Fluids 8, 1295-1299.

[81] Turland, B. D. and Scheuer, P. A. G. (1976). "Instabilities of Kelvin-Helmholtz type for relativistic streaming." Monthly Notices Roy. Astronom. Soc. 176, 421-441.

[82] Weissman, M. A. (1979). "Nonlinear Wave Packets in the Kelvin-Helmholtz instability." Philos. Trans. Roy. Soc. London 290, 639.

[83] Whitham, G. B. (1974). "Linear and Nonlinear Waves." New Jersey: Prentice Hall.

[84] Winant, C. D., and Browand, F. K. (1974). "Vortex pairing: The mechanism of turbulent mixinglayer growth." Journal Fluid Mech. 63, 237-255.

[85] Wollkind, D. J., Manoranjann, V. S., and Zhang, L. (1994). "Weakly Nonlinear Stability Analyses of Prototype Reaction Diffusion Model Equations." SIAM Review 36, 176. 